\title{
1 RBL1 (p107) functions as tumor suppressor in glioblastoma 2 and small-cell pancreatic neuroendocrine carcinoma
}

3 Thomas Naert ${ }^{1,2, \&}$, Dionysia Dimitrakopoulou ${ }^{1,2, \&}$, Dieter Tulkens ${ }^{1,2}$, Suzan Demuynck ${ }^{1}$, Rivka

4 Noelanders $^{1,2}$, Liza Eeckhout ${ }^{1}$, Gert van Isterdael ${ }^{3}$, Dieter Deforce ${ }^{4}$, Christian Vanhove ${ }^{2,5}$, Jo Van

5 Dorpe $^{2,6}$, David Creytens ${ }^{2,6}$, Kris Vleminckx ${ }^{1,2,7, \S}$.

$6{ }^{1}$ Department of Biomedical Molecular Biology, Ghent University, Ghent, Belgium

$7 \quad{ }^{2}$ Cancer Research Institute Ghent, Ghent, Belgium

$8{ }^{3}$ Inflammation Research Center, VIB, Ghent, Belgium

$9{ }^{4}$ Laboratory for Pharmaceutical Biotechnology, Ghent University, B-9000 Ghent, Belgium

$10{ }^{5}$ Infinity lab, Ghent University Hospital, Ghent, Belgium

$11{ }^{6}$ Department of Pathology, Ghent University and Ghent University Hospital, Ghent, Belgium

$12{ }^{7}$ Center for Medical Genetics, Ghent University, Ghent, Belgium

13 \& Contributed equally to this work

$14{ }^{\S}$ Corresponding author

15 Kris Vleminckx, Ph.D.

16 Dept. for Biomedical Molecular Biology

17 Ghent University

18 Technologiepark 927

19 B-9052 Ghent (Zwijnaarde)

20 Tel +32-9-33-13760, Fax +32-9-221 76 73,

21 E-mail : kris.vleminckx@irc.UGent.be

22

23 Competing interests

24 The authors declare no competing interests. 


\section{Abstract}

28 Alterations of the retinoblastoma and/or the p53 signaling network are associated with specific cancers 29 such as high-grade astrocytoma/glioblastoma, small cell lung cancer (SCLC), choroid plexus tumors and 30 small-cell pancreatic neuroendocrine carcinoma (SC-PaNEC). However, the intricate functional 31 compensation between RB1 and the related pocket proteins RBL1/p107 and RBL2/p130 in suppressing 32 tumorigenesis remains poorly understood. Here we performed lineage-restricted parallel inactivation of $33 r b 1$ and rb/1 by multiplex CRISPR/Cas9 genome editing in the true diploid Xenopus tropicalis to gain 34 insight into these in vivo compensatory mechanisms. We show that while $r b 1$ inactivation is sufficient to 35 induce choroid plexus papilloma, combined $r b 1$ and $r b / 1$ inactivation is required and sufficient to drive 36 SC-PaNEC, retinoblastoma and astrocytoma. Further, using a novel Li-Fraumeni syndrome-mimicking 37 tp53 mutant $X$. tropicalis line, we demonstrate increased malignancy of retinoblastoma-mutant neural 38 malignancies upon concomitant inactivation of tp53. Interestingly, although clinical SC-PaNEC samples 39 are characterized by abnormal p53 expression or localization, in the current experimental models, the 40 tp53 status had little effect on the establishment and growth of SC-PaNEC, but may rather be essential 41 for maintaining chromosomal stability. SCLC was only rarely observed in our experimental set-up, 42 indicating requirement of additional or alternative oncogenic insults. In conclusion, we used CRISPR/Cas9 43 to delineate the tumor suppressor properties of Rbl1 and generate new insights in functional 44 compensation within the retinoblastoma protein family in suppressing pancreatic and specific neural 45 cancers.

\section{$46 \quad$ Keywords}

47 p107, p53, CRISPR/Cas9, Pancreatic neuroendocrine carcinoma, choroid plexus, retinoblastoma, Xenopus 48 tropicalis 


\section{Introduction}

51 The interplay of the signaling networks controlling cell cycle (e.g. Retinoblastoma (RB)) and cell death 52 (e.g. p53) in suppressing the development of cancers including, amongst others, glioblastoma, choroid 53 plexus carcinoma, pancreatic neuroendocrine carcinoma and small-cell lung cancer was previously 54 demonstrated by clinical and animal modeling studies ${ }^{1-6}$. Unsatisfactory, the median survival prospects 55 for patients diagnosed with p53 and RB1 deficient cancers are extremely dismal, e.g. for small cell lung 56 cancer (SCLC) (stage IV: 8-10 months ${ }^{7}$ ), small cell pancreatic neuroendocrine carcinoma (SC-PaNEC) (11 57 months ${ }^{8}$ ), choroid plexus tumors (tp53 ${ }^{\text {mutated }}$ WHO grade III: 2-4 months ${ }^{9}$ ) and glioblastoma (12-18 months ${ }^{10}$ ). As such, continued generation of novel and short latency preclinical models for these highly aggressive cancers remains necessary to fuel rational design of molecular targeted drug therapies.

Intriguingly, the RB signaling network entails a family of three pocket proteins (RB1, RBL1, RBL2), which in union tightly regulate $\mathrm{G} 1 / \mathrm{S}$ cell cycle progression, and whose differential expression may underlie cell-type specific functional compensation in suppressing tumorigenesis ${ }^{11,12}$. An example of this compensation occurs in the mouse and Xenopus retina where RB1-deficiency fails to initiate retinoblastoma, and simultaneous inactivation of $R b / 1$ (p107) is required to initiate tumorigenesis ${ }^{13-15}$. In an alternative case, high-grade astrocytoma and choroid plexus tumors have been previously induced in murine models by overexpression of $\mathrm{T}_{121}$, a truncated SV40 T antigen mutant that binds and inhibits RB1, RBL1 and RBL2 proteins ${ }^{16,17}$. In order to investigate the selective tumor suppressor properties of the Rbl1 protein, thus dissecting compensation on a genetic level, we performed concomitant inactivation of the rb1 and rb/1 tumor suppressor genes, leaving rb/2 unaltered. For this we used CRISPR/Cas9 gRNA ribonucleoproteins (RNPs) delivered by microinjection in embryos of Xenopus tropicalis, an aquatic amphibian with an unique true diploid genome. By targeting the injections to specific blastomeres we were able to modify the retinoblastoma signaling network in different tissue lineages, including the neural and pancreatic lineages ${ }^{18,19}$. As such, we aimed to obtain a deeper understanding of tissuespecific compensation between RB1 and RBL1 in cancer development ${ }^{20}$.

Next to manipulating the RB pathway, we also generated a tp53 knockout Xenopus line as a stable genetic background with a twofold aim. We wanted to explore whether Li-Fraumeni syndrome (LFS) could be recapitulated in Xenopus tropicalis. In addition, we wanted to investigate whether tp53 deficiency sensitizes the animals to tumor initiation or progression upon additional genetic oncogenic insults, as has been extensively documented in the mouse. LFS is a cancer syndrome defined by either incapacitating germline TP53 mutations or de novo mutations occurring during early embryogenesis ${ }^{21,22}$.

81 LFS patients are at risk for development of (osteo-)sarcomas, central nervous system (CNS) tumors, 
82 breast cancer and adrenocortical carcinoma, as well as other less frequent cancers ${ }^{23}$. Previously, $T p 53$

83 knockout mouse models have been generated and were shown to be prone to the development of a 84 variety of cancers, most frequently lymphomas and sarcomas ${ }^{24-26}$. Additionally, LFS was modeled in tp53

85 mutant zebrafish spontaneously developing malignant peripheral nerve sheet tumors (MPNST) and angiosarcoma, next to other tumor types ${ }^{27,28}$.

As mentioned before, p53 is an established gatekeeper for numerous tumor types. Hence, experimental oncogenic insults, either genetic or environmental, are frequently employed in a $T p 53$ mutant background because it increases tumor penetrance and reduces tumor latency. As such, we also performed parallel perturbation of the retinoblastoma and p53 signaling network, by multiplexing $r b 1$, rbl1 CRISPR/Cas9 and inactivating tp53 by either CRISPR/Cas9 multiplexing or breeding efforts. We investigated whether sensitizing Xenopus tropicalis to cancer development by perturbing p53 functions could alter the tumor spectrum occurring upon perturbations in the retinoblastoma signaling network. In this regard, previous work also described that concomitant perturbation of the retinoblastoma and p53 signaling network generates chromosomal instability (CIN), possibly sensitizing to cancer development ${ }^{29-}$ 31.

By our in vivo gene editing strategy we identify novel cell-type specific requirements for inactivation of rb/1, in order to bypass compensation in the native and immunocompetent Xenopus brain and pancreas upon inactivation of $r b 1$, across different tp53 genotypes. By combinatorial CRISPR/Cas9 editing of $r b 1$ and $r b / 1$ we demonstrate highly penetrant development of SC-PaNEC, glioblastoma, choroid plexus tumors, next to other cancer types. Interestingly, we provide genetic evidence establishing rb/1 as a genuine tumor suppressor in SC-PaNEC and glioblastoma.

We believe our novel genetic $X$. tropicalis genetic cancer models have the potential to generate important molecular insights and could emerge as promising preclinical cancer models ${ }^{32}$. Namely, these mice models, mainly due to the relative ease of CRISPR/Cas9 delivery and the short latency and high penetrance of cancer development ${ }^{33}$.

\section{Results}


111 In order to generate a tp53 mutant $X$. tropicalis line, we unilaterally injected tp53 coding region 1

$112\left(t p 53^{\mathrm{cr} 1}\right)$ gRNA precomplexed with recombinant Cas9 protein in two-cell $X$. tropicalis embryos. Mosaic

113 mutant F0 animals, further called crispants, were raised until sexual maturity and out-crossed with wild-

114 type animals. Upon targeted amplicon sequencing and BATCH-GE analysis ${ }^{34}$, we selected two types of F1 $115 t p 53$ heterozygotes, each with a distinct variant of a $\Delta 4$ deletion ( $t p 53^{\Delta 4 \mathrm{var} 1 /+}$ and $t p 53^{\Delta 4 \mathrm{var} /++}$ ) (Table S1A).

116 Both of these deletions are expected to give rise to nonsense-mediated decay since due to the frame-

117 shifting deletion, the pre-mRNA contains a premature stop codon ( $t p 53^{1685 T O P}$ ). These heterozygote

118 mutants (F1) were subsequently intercrossed $\left(\operatorname{tp} 53^{\Delta 4 \mathrm{var} 1 /+} \times \operatorname{tp} 53^{\Delta 4 \mathrm{var} 2 /+}\right)$ to obtain $\mathrm{F} 2$ homozygous mutant

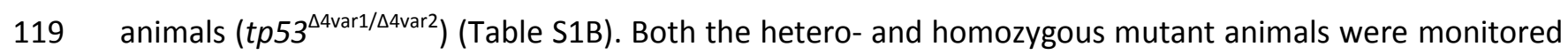

120 for morbidity and euthanized at ethical endpoint defined by distended abdomen reminiscent of ascites,

121 lethargy, or decreasing body weight. Of note, $X$. tropicalis has a significantly longer lifespan than murine

122 animal models and can reach over ten years of age in experimental housing conditions.

123 Kaplan-Meier analysis over a period of 900 days revealed faster occurrence of morbidity in two 124 clutches of $\operatorname{tp} 53^{\Delta 4 \mathrm{var} 1 / \Delta 4 \mathrm{var} 2}(\mathrm{~F} 2)$ nullizygous animals when compared to tp53 heterozygotes (F1) $(p<0.01$;

125 Table S2A) (Fig. 1A). We investigated moribund mutant $X$. tropicalis by $X$-ray techniques, revealing 126 calcified ectopic structures in $66 \%(n=3)$ of F1 tp53 heterozygous animals (Fig. 1B).

In order to investigate the possible presence of hematological malignancy, we performed immunohistological analysis of the spleen in moribund tp53 mutant animals. In the wild type Xenopus spleen, $\mathrm{CD}^{+}$cells organize in a ring-like structure surrounding the white pulp, which contains the B-cells and displays pronounced PCNA immunoreactivity (Fig. 1C-left) ${ }^{35}$. However, this $\mathrm{CD}^{+}{ }^{+}$ring-like structure was disrupted in $75 \%(n=8)$ of the moribund $t p 53^{\Delta 4 v a r 1 / \Delta 4 v a r 2}$ animals (Fig. 1C-right; Fig. 1D). In $25 \%(n=8)$ of these animals we also observed that PCNA immunoreactivity expanded into red pulp areas not contiguous with CD3 immunoreactivity. As such, we conclude that the observed splenic architectural disruptions are reflecting the presence of different hematologic malignancies either or not of the T-cell lineage, which in one case lead to profound cleaved caspase-3 immunostaining in splenic white pulp (Fig. S1A). Interestingly, both malignancies of the B- and T-cell lineages have been described in murine tp53 mutant animals models ${ }^{36}$.

In order to further investigate the presence of T-cell-related hematological malignancy in 140 toe clipping and subjected to flow cytometry to investigate the proportion of cells of the T-cell lineage. 141 Enrichment of $\mathrm{CD}^{+}$(cytoplasmic or membrane located) and $\mathrm{CD} 8^{+}$cells could be demonstrated in one out 142 of six of the non-moribund animals (Fig. 1F; Fig. S2-3). An increased leukocyte count was subsequently 
validated by Natt-Herrick's method in blood obtained by cardiac bleed in this animal, when compared to an age-matched control ( $p<0.01$; Table S2B) (Fig. 1E; Fig. S1B). Furthermore, in this $t p 53^{-\%}$ animal, CD3 ${ }^{+}$ cells were also disorganized in the spleen and diffuse dissemination of $\mathrm{CD}^{+}$T-lymphocytes could be observed in the liver (Fig. 1G; Fig. S1C). Furthermore, we also observed the presence of liposarcoma in one $t p 53^{--}$animal (Fig. S1D).

Finally, one moribund tp53 heterozygous animal presented with a large intraperitoneal lobular neoplasm, classified by histopathology as a high-grade undifferentiated spindled and round cell sarcoma with profound PCNA proliferation marker immunoreactivity (Fig. 1H).

Taken together, we demonstrate development of a LFS-type tumor spectrum with hematological malignancy and sarcoma in tp53 mutant $X$. tropicalis, in line with well-characterized Tp53 mutant mouse models ${ }^{24,25}$. The latency to neoplasm-related morbidity (>1 year) is relatively long in the tp53 mutant $X$. tropicalis, but can likely be attributed to the relative long lifespan ( 10 years) of Xenopus. In any case, these animals constitute a valuable platform for evaluating the role of the $t p 53$ tumor suppressor gene inactivation in combination with other oncogenic insults. Hence, we continued on to target retinoblastoma-family tumor suppressor genes in this tp53 mutant line. editing

In order to investigate the functional consequences of inactivating $r b 1$ and $r b / 1$, under differential tp53 genetic backgrounds, we performed multiplex CRISPR/Cas9-mediated inactivation of these genes in tp53 mutant and wild-type $X$. tropicalis. Note that the rb1 and rb/1 gRNAs used throughout this study have previously been reported to induce retinoblastoma in $X$. tropicalis ${ }^{14}$. These gRNAs were shown to induce retinoblastoma independent from CRISPR/Cas9 off-target effects, as in that study another pair of $r b 1 / r b / 1$ gRNAs with a differential genomic targeting site similarly induced this tumor type. For targeting $t p 53$, we also included a gRNA that was different from the one used to make the KO line, i.e. $t p 53^{\text {coding }}$ region 2 or $t p 53^{\text {cr2 }}$.

We intercrossed $t p 53^{\Delta 4 v a r 1 /+}$ with $t p 53^{\Delta 4 v a r 2 /+}$, generating embryos with either $t p 53^{+/+}, \operatorname{tp} 53^{\Delta 4 v a r 1 /+}$, $t p 53^{\Delta 4 v a r 2 /+}$ or $t p 53^{\Delta 4 v a r 1 / \Delta 4 v a r 2}$ genotype, while simultaneously intercrossing wild-type (WT) $X$. tropicalis to obtain WT embryos. We subsequently injected tp53 mutant or WT embryos in the eight-cell stage in a single vegetal-dorsal blastomere with distinct combinations of tumor suppressor-targeting CRISPR/Cas9 RNPs (Fig. 2A). As thus we performed primarily genome editing of the anterior endoderm, including the pancreas. Genome editing was confirmed by targeted amplicon sequencing and BATCH-GE analysis 
174 (Table S1C). Animals were euthanized when presenting with distended abdomen, lethargy and

175 decreasing bodyweight or at end-of-experiment (97 days). Necropsy revealed no gross pancreatic 176 abnormalities in animals resulting from $r b 1$ CRISPR/Cas9 injection in embryos obtained from a $t p 53^{\Delta 4 v a r 1 /+}$

$177 \times$ tp53 $3^{\Delta 4 v a r 2 /+}$ intercross (hereafter termed single mosaic knockout (smKO) at 97 days ( $\left.n=13\right)$. However, we 178 observed pancreatic dysmorphology in $86 \%(n=29)$ of the animals resulting from multiplexed $r b 1 / r b / 1$ 179 CRISPR/Cas9 injection in embryos obtained from the same intercross (hereafter termed double mosaic 180 knockout (dmKO)) and in $77 \%(\mathrm{n}=22)$ of the animals resulting from $\mathrm{rb1} / \mathrm{rb} / 1 / \mathrm{tp} 53^{\mathrm{cr} 2}$ CRISPR/Cas9 injection 181 in WT embryos (hereafter termed triple mosaic knockout (tmKO)). Both for dmKO and tmKO, these 182 pancreatic dysmorphologies were observed prior to the 70 day timepoint (Fig. 2B-C; Table S2C). 183 Histologically, we observed cellular, poorly differentiated malignant tumors with a 'small blue round cell' 184 morphology, cellular pleomorphism, high mitotic activity and foci of hemorrhage and necrosis, 185 compatible with the histology of SC-PaNEC (Fig. 2D) ${ }^{37}$. A high proliferative index was demonstrated further by both anti-proliferating cell nuclear antigen (PCNA) and anti-Ser10 phosphorylated histone $\mathrm{H} 3$ (pHH3) immunostaining (Fig. 2E).

Tp53 mutations are not essential for small-cell pancreatic neuroendocrine carcinoma (SC-PaNEC)

\section{formation}

The near-complete penetrance of SC-PaNEC development in dmKO animals, which are derived from a $t p 53^{+/}$intercross wherein $75 \%$ of the individuals are expected to have at least one wild-type copy of $t p 53$, provided preliminary evidence that $t p 53$ does not have an essential gatekeeper function in preventing SC-PaNEC tumorigenesis. In order to validate this further, we first performed genotyping of tp53 in each dmKO animal and showed that indeed the clutch followed expected Mendelian ratios $\left(\mathrm{H}_{0}=\right.$ non-Mendelian; $p=0.63$ ) (Table S1D and S2D) ${ }^{38}$. We observed no correlation between tp53 genotype and incidence of SC-PaNEC development ( $p>0.05$; Table S2E) or between SC-PaNEC size and tp53 genotype ( $p>0.05$; Fig. S4A; Table S2E). Furthermore, no obvious differences in cell death between tp53/- or $t p 53^{+/+}$SC-PaNECs could be demonstrated by TUNEL staining (Fig. S4B). In fact, SC-PaNEC cells do not show TUNEL positivity, in stark contrast to the immediately surrounding normal pancreatic cells, possible due to cellular stress from the adjacent neoplasm. Finally, investigation of SC-PaNEC proliferation characteristics across different tp53 genotypes, by quantification of pHH3 positive cells in tissue sections, did not reveal any genotype-specific differences (Fig. S4C; Table S2F).

Interestingly, the genetic make-up of the $t p 53$ nullizygous mutant animals, with a distinct $\Delta 4$ variant 
capture microdissection (LCM) and tp53 genotyping of SC-PaNECs from the dmKO. We observed chromosomal instability (aneuploidy) involving chr3.p in 66\% $(n=3)$ of $t p 53^{-\%}$ SC-PaNECs in the dmKO setup (Fig. 2F; Table S1E; Fig. S5). In contrast, $t p 53^{+/-}$SC-PaNEC $(n=3)$ in the dmKO retained the expected allelic ratios, demonstrating that $t p 53 \mathrm{LOH}$ is not occurring and that SC-PaNECs can initiate without biallelic germline mutations in tp53 (Fig. 2F; Fig. 2G - blue ovals). Confirming this further, we showed by similar LCM and genotyping methodology that two tumors in the tmKO setup $(n=7)$ developed without biallelic CRISPR/Cas9-mediated inactivation of the tp53 gene (red ovals - Fig. 2G) . Taken together, we found that inactivation of tp53 is not essential for SC-PaNEC tumor initiation, but provide, albeit preliminary, evidence that its inactivation might drive genomic instability ${ }^{31}$.

\section{Rbl1 functions as a tumor suppressor in SC-PaNEC}

215 Given that we could not detect any development of SC-PaNECs in smKO ( $r b 1$ mosaic mutant) animals, but SC-PaNECs were readily detected in $r b 1 / r b / 1$ double mosaic mutants (dmKO and tmKO animals), we hypothesized that $r b / 1$ is functioning as a genuine tumor suppressor gene in SC-PaNEC.

Previously, multiplex CRISPR/Cas9 technology has been described as a platform enabling in vivo

functional interrogation of genes in pancreatic ductal carcinoma ${ }^{39,40}$. Namely, genes functioning as genuine tumor suppressors are found to be mutated in tumors due to positive selection for inactivating genomic alterations. We had also previously demonstrated similar positive selection pressure on combined $r b 1$ and $r b / 1$ inactivation in a pediatric $X$. tropicalis retinoblastoma model ${ }^{14}$.

We performed LCM of SC-PaNECs, followed by both $r b 1$ and $r b / 1$ genotyping. We could demonstrate fully penetrant positive selection pressure for both $r b 1$ and $r b / 1$ mutations in SC-PaNECs from tmKO $(100 \% ; n=7)$ animals (Table S1F). Similarly, positive selection for simultaneous $r b 1$ and $r b / 1$ mutations was found in SC-PaNECs from dmKO (100\%; $\mathrm{n}=6$ ) animals (Fig. 2G). Overall, our data shows that $r b 1$ inactivation alone, irrespective of tp53 genotype, is not sufficient to initiate SC-PaNEC in $X$. tropicalis.

228 This appears to be due to redundancy with the rb/1 gene, since we demonstrate that inactivation of the retinoblastoma pathway by multiplex $r b 1 / r b / 1$ inactivation results in highly penetrant SC-PaNEC formation with short latency.

Given the non-necessity for tp53 inactivation in SC-PaNEC development, we further aimed to provide 233 a proof-of-principle that p53 can indeed have tumor suppressive functions in other $X$. tropicalis Rb1234 deficient cancers. We performed ectoderm-specific CRISPR/Cas9-mediated genome editing of rb1 and 
rbl1 in embryos obtained from a tp53 $3^{\Delta 4 v a r 1 /+}$ and tp $53^{\Delta 4 v a r 2 /+}$ intercross, via injection of an animal-dorsal blastomere (Fig. 3A) (Table S1G). Animals were euthanized at metamorphic climax (69 days post-

237 injection) and necropsy revealed that $75 \%(n=16)$ of the animals presented with externally visible retinoblastoma development (Fig. 3B). Furthermore, $44 \%(n=16)$ of the animals had excessive black skin pigmentation, indicative of a disturbed neuroendocrine reflex in the pituitary or hypothalamus for the regulation of pigment dispersion in dermal melanophores (Fig. 3B-C) ${ }^{41}$. This prompted us to investigate the animals for CNS abnormalities. Genotyping of $t p 53$ was performed by targeted amplicon sequencing for each animal within the setup (Table $\mathrm{S} 1 \mathrm{H})$. Histopathology revealed presence of retinoblastoma and

243 brain-located poorly differentiated and highly malignant small blue round cell tumors (SBRCTs), in 244 respectively $75 \%$ and $43 \%(n=16)$ of the animals, closely recapitulating previously reported 245 retinoblastoma incidences in $r b 1 / r b / 1$ crispants (Fig. 3D) ${ }^{14}$. The SBRCTs (Fig. 3E - white arrows) likely 246 correspond to either invasive retinoblastoma, pinealoblastoma (trilateral retinoblastoma) or possibly 247 medulloblastoma ${ }^{42}$. In any case, SBRCTs presented with aggressive growth characteristics as indicated by 248 PCNA immunoreactivity (Fig. S6A). Comparison of retinoblastoma and SBRCTs incidences across tp53 249 genotype did not reveal any clear correlation ( $p>0.05$; Table S2G). Furthermore, in line with previously 250 published studies in the mouse, histopathological hallmarks of $r b 1 / \mathrm{rb} / 1$ inactivated retinoblastoma did 251 not change upon tp53 pathway inactivation ${ }^{43}$. Interestingly however, we also observed penetrant 252 induction of choroid plexus neoplasms (Fig. 3E - black arrow) and glioblastoma (Fig. 3E - red arrow) in 253 the CNS of $\operatorname{tp} 53^{\Delta 4 \mathrm{var} 1 / \Delta 4 \mathrm{var} 2}$ animals (Fig. S6B). Therefore, we first performed a differential analysis 254 comparing the grade and incidence of choroid plexus tumors across tp53 genotypes.

\section{Tp53 mutational status underlies retinoblastoma-mutant choroid plexus tumor progression}

256 Having observed the presence of choroid plexus tumors (CPT) in rb1 and rb/1 double crispant tadpoles, 257 we aimed to investigate whether, in line with the clinical data, tp53 inactivation is linked with an 258 increased CPT grade ${ }^{9}$. For this, anonymized sections from choroid plexi $(n=15)$ were analyzed by a 259 pathologist and classified according to clinical WHO grading criteria in one of three categories: normal, 260 grade 1 (G1) (choroid plexus papilloma) or >G1 (atypical choroid plexus papilloma and choroid plexus 261 carcinoma) ${ }^{44}$. Normal amphibian choroid plexus closely resembles the mammalian structural 262 organization, where ependymal cells, capillaries and pia mater can be readily discriminated at high 263 magnification (Fig. 4A). G1 neoplasms were characterized by cellular disarray, (pseudo-)stratification and 264 loss of polarization (Fig. 4A -arrow), while mitotic figures were relatively rare. In higher grade (>G1) 265 neoplasms, pronounced cellular disarray and severely increased mitotic figures could be observed. Clear 
continuity between normal choroid plexus (CP) and neoplasms was also demonstrated (Fig. 4B). By this subclassification, a correlation between tp53 genotype and choroid plexus neoplasm grade could be demonstrated ( $p<0.01$; Table S2H) (Fig. 4C). Furthermore, immunofluorescence, in a $t p 53^{\Delta 4 v a r 1 / \Delta 4 v a r 2}$ animal, further revealed profound PCNA immunoreactivity in a >Grade 1 neoplasm, demonstrating high proliferative activity (Fig. 4D - white arrow).

Finally, we performed LCM and targeted amplicon sequencing of the tp53 locus in choroid plexus tumors and control tissue (Fig S7; Table S1I). As described previously for PaNECs, the genetic make-up of

273 the tp53 homozygous mutant animals allows assessment of genomic instability on chr3.p. In

$274 \operatorname{tp} 53^{\Delta 4 \mathrm{var} 1 / \Delta 4 \mathrm{var2}}$, the $>\mathrm{G} 1$ choroid plexus neoplasms exhibited either loss of compound heterozygosity or

275 hyperploidy of one $\Delta 4$-variant, while control tissue (normal brain, bone) exhibited the expected normal 276 allelic ratios (50\% each variant) (Fig. 4E - left). Furthermore, in a high-grade CP lesion in a heterozygous 277 tp53 mutant animal, we could demonstrate LOH of the remaining WT tp53 allele (Fig. 4E - right). We 278 found lack of positive selection pressure for $r b / 1$ mutations, as we observed that the $r b / 1$ allele remained 279 wild-type in tp53 nullizygous CPTs $(n=3)$ (Table S1J). As such, rb/1 does not appear to function as a tumor suppressor gene in CPTs.

Rb/1 functions as a tumor suppressor in glioma, while tp53 inactivation underlies malignant progression

Further analyzing the CNS of $r b 1$ and $r b / 1$ multiplex CRISPR/Cas9-engineered animals obtained from a $\operatorname{tp} 53^{\Delta 4 v a r 1 /+}$ and $\operatorname{tp} 53^{\Delta 4 v a r 2 /+}$ intercross, we also observed the occurrence of glioma with pronounced intratumoral heterogeneity in $81 \%(n=16)$ of these animals. A significant correlation between tp53 genotype and glioma grade was present, with high-grade glioblastoma lesions only seen in $t p 53^{\Delta 4 v a r 1 / \Delta 4 v a r 2}$ animals $(p<0.001)$ (Fig. 5A,B; Fig. S8A) (Table S2I). In line with murine Rb1-mutant glioblastoma, highgrade lesions were characterized by the presence of pleomorphic giant cells, mitotic defects and massive nuclear aneuploidy (Fig. 5A, B) (Supplemental Movie 1) ${ }^{5,45}$. In depth microscopic analysis of these nuclear abnormalities showed some to be interconnected across distances spanning tens of micrometers (Supplemental Movie 2). In the higher grade lesions, the giant cells were also interspersed with smaller rounder cells with dense nuclei. Here, both cell-types possessed a high proliferative index as demonstrated by PCNA immunostaining (Fig. 5E). Furthermore, we showed heterogeneous immunoreactivity to a GFAP antibody throughout these actively cycling cells, in line with literature and validating astrocytic origin (Fig. S8C) ${ }^{10}$. Glioblastoma cells were observed predominantly in areas contiguous with normal proliferative areas (subventricular zone), especially in the forebrain (Fig. 5B-C), 
298

299

300

301

302

303

304

305

306

307

308

309

310

but also in regions not immediately spatially associated with such proliferative zones. Areas of pseudopallisading necrosis or hemorrhaging were absent. We found profound EZH2 immunoreactivity in forebrain lesions associated with the SVZ, pointing to a proneural glioblastoma, at least at this anatomical location (Fig. 5E; Fig. S8B) ${ }^{46,47}$. Unfortunately, further attempts to perform glioblastoma subtyping were unsuccessful, due to lack of suitable antibodies in Xenopus tropicalis.

The presence of astrocytoma in animals exclusively mutant in $r b 1$ and $r b / 1$ (Fig. 5A, D), pruned us to investigate whether $r b / 1$ compensates for the loss of $r b 1$, under $t p 53$ wild-type conditions. For this, we performed a retrospective analysis of brain sections from our previously published retinoblastoma study in animals unilaterally targeted at the two-cell stage with $r b 1$ and $r b / 1$ gRNA ${ }^{14}$. We detected the presence of astrocytoma in $100 \%(n=9)$ of the animals injected with the rb1/rb/1 gRNAs (Fig. S9). Astrocytoma was also found in animals injected with another pair of $r b 1 / r b / 1$ gRNA, ruling out potential CRISPR/Cas9 off-target effects as contributing to the observed phenotype. Interestingly, some astrocytoma lesions in this cohort had spontaneously progressed to high-grade glioblastoma, demonstrating that rb1/rbl1-deficient glioma progression is indeed possible in vivo (Fig. S9 - black arrow).

We further wanted to investigate whether the requirement for rb/1 inactivating mutations can be overruled by additional oncogenic insults. Co-inactivation of pten was chosen as this gene has been widely linked to glioblastoma malignancy ${ }^{5,48}$. Therefore, we performed ectoderm-specific CRISPR/Cas9mediated genome editing of $r b 1$ and $p t e n^{c r 1}$ in embryos obtained from a tp53 $3^{\Delta 4 v a r 1 /+}$ and $\operatorname{tp} 53^{\Delta 4 v a r 1 /+}$ intercross, thus leaving rb/1 intact (Table S1K). Animals were euthanized at day 42 and histopathology revealed absence of any glioma $(0 \% ; n=14)$ in either of the three tp53 genotypes, including four $t p 53^{\Delta 4 v a r 1 / \Delta 4 v a r 1}$ animals (Fig. 5D, Table S1L). In contrast, animals injected with $r b 1, r b / 1, t p 53^{c r 2}$ and $p t e n^{c r 2}$ in the ectodermal lineage demonstrated complete penetrance in high-grade glioblastoma development (100\%; $n=13$ ) at day 42 (Fig. 5C-D; Table S1M).

Collectively, our experiments provide the direct genetic evidence that $r b / 1$ functions as a tumor suppressor compensating for loss of rb1 in rb1-mutant astrocytes and that concomitant inactivation of $r b 1 / r b / 1$ is sufficient for glioma initiation. Further, and in line with literature, we demonstrate that $\operatorname{tp} 53$ inactivation facilitates progression of retinoblastoma-deficient glioma to high-grade glioblastoma ${ }^{5}$.

\section{Discussion}

Members of the retinoblastoma pocket protein family (RB1, RBL1, RBL2) play a pivotal role in the control of cellular proliferation and their direct inactivation by mutational events has been previously 
329 implicated in cancers, such as small-cell lung carcinoma (SCLC) and retinoblastoma ${ }^{13,49}$. However, 330 contemporary approaches for understanding and modeling cancer has conceptualized that signaling 331 networks can be disturbed at diverse nodes, converging on similar downstream phenotypical 332 consequences. For instance, within the RB signaling network, the ability of RB1 and RBL1 to regulate the 333 cell cycle can be bypassed by events altering their common upstream cyclin dependent kinase regulators 334 (i.e CDK4, CDKN2A/p16). Such direct genetic deregulation of the RB signaling network by either CDK4 335 amplification, or inactivation of $C D K N 2 A / B$ or $R B 1$, had been previously shown in human SC-PaNEC and 336 glioblastoma ${ }^{4,50-52}$. In contrast, direct genetic inactivation of $R B L 1$ (p107) remains rare $(<0.5 \%)$ in clinical 337 glioma samples. However, reanalyzing cancer genomic data for combined cohort of 8 glioma studies 338 validated mutual exclusivity between $C D N K 2 A / B$ and $C D K 4$ alterations $(p<0.001)$, but established 339 significant co-occurrence of $R B 1$ and $R B L 1$ alterations $(p=0.034)$ (Table $S 3)^{53,54}$. As such we postulate 340 that directly inactivating $r b 1$ and $r b / 1$ could contribute to cancer initiation, similar to indirect 341 simultaneous inactivation of RB1 and RBL1 protein function due to prolonged phosphorylation by 342 upstream Cyclin/CDK complexes. This hypothesis is further supported by reports that RBL1 is 343 downregulated in glioma tumors and cell lines ${ }^{55}$. Furthermore, $R b 1$ deficiency alone fails to initiate 344 tumorigenesis in astrocytes, while transgenic in vivo expression of a truncated SV40 large $T$ antigen ( $\left.T_{121}\right)$, 345 which binds and inhibits all three retinoblastoma family members, is sufficient for tumorigenesis ${ }^{56}$. 346 Additionally, a $R b 1 / R b / 1 / T p 53 / P t e n$ conditional murine glioblastoma model was previously generated 347 and used as a platform for preclinical studies ${ }^{47,57}$. However because the full characterization of this line 348 is, up to this date, not reported we cannot delineate the exact impact of Rb/1 inactivation. Finally, it was 349 shown that Rb/1-deficient mice exhibit expanded neural stem cell population and impaired commitment 350 of neuronal progenitors to a neuronal fate, showing previously underappreciated functions of Rbl1 ${ }^{58,59}$. 351 In fact, to our knowledge the definitive genetic in vivo elucidation of RBL1 as a glioma tumor suppressor 352 has not been reported before. Here we showed that rb/1 inactivation is a cooperative event in the rapid 353 establishment of glioma in immunocompetent rb1-deficient Xenopus tropicalis. Furthermore, we believe 354 that while direct genetic inactivation of the $R B L 1$ gene is rare $(0.3 \%)$ in glioma, additional mechanisms 355 are at play in which the RBL1 protein could in fact be a major player in suppressing tumorigenesis, but 356 has had its role previously seen minimized due to its indirect inactivation at a functional level.

357 We also demonstrate that concomitant inactivation of $r b 1$ and $r b / 1$ is sufficient to induce SC-PaNEC. 358 Unfortunately, as a consequence of the low incidence of SC-PaNEC, no large sets of whole-exome or 359 whole-genome sequencing data are available to address the status of the RBL1 gene in clinical SC-PaNEC. 360 Nevertheless, previous studies already suggested functional compensation between pocket proteins in 
361 the endocrine pancreas, where inactivation of $R b 1$ has little effect on $\beta$-cell replication, while 362 concomitant $R b 1 / R b / 1$ and $R b 1 / R b / 2$ inactivation has profound impact on proliferation and apoptosis ${ }^{60-}$ $363 \quad 62$.

364 In contrast to these newly discovered rbl1 tumor suppressor roles, we also show that choroid plexus tumorigenesis does not critically depend on rb/1 inactivation. Additionally, while we had previously and incidentally discovered a small cell lung carcinoma in a $r b 1 /$ rb/1 crispant ${ }^{14}$, we fail to identify these in larger cohorts of $r b 1 / r b / 1$ crispants, across different tp53 genotypes. Interestingly, this is in line with mice modeling data placing $R b / 2$ ( $p 130)$, and not $R b / 1$, at the center of tumor suppression in $r b 1$-deficient endocrine lung cells ${ }^{49}$. This reflects, cell-type specific responses to inactivation of distinct retinoblastoma network elements, whose interplay could be further dissected using CRISPR/Cas9 in Xenopus ${ }^{11}$.

Furthermore, we also co-interfered with the cell signaling death network by targeting tp53, in order to gain insight in how co-occurring p53 aberrations influences rb1/rb/1-deficient cancers. In contrast to 373 the accepted paradigm that concomitant p53 and retinoblastoma signaling network abnormalities are required to initiate SC-PaNEC, we demonstrate unexpected low impact of tp53 inactivation in $r b 1 / r b / 1$ inactivated SC-PaNEC ${ }^{2}$. In line with our results, the implication of p53 in clinical SC-PaNEC is predominated by abnormal immunostaining of the $\mathrm{p} 53$ protein, rather than absence of reactivity, which would be indicative for p53 loss-of-expression ${ }^{63,64}$. We speculate that TP53 mutational events could be dispensable in early SC-PaNEC tumorigenesis, but that TP53 alterations and their consequent chromosomal instability could eventually leads to highly malignant and metastatic SC-PaNEC ${ }^{29}$. Unfortunately SC-PaNEC is considered to be unresectable and mostly metastatic at the time of diagnosis and hence patients are almost immediately treated with chemotherapy, precluding clinical assessment of TP53 alterations in early stages of SC-PaNEC development. Regrettably, the tumors in our Xenopus model are too fast growing, leading to morbidity and ethical endpoint prior to metastasis formation, impeding assessment of this hypothesis.

In contrast to the above and closely recapitulating the known roles of $\mathrm{p} 53$, we demonstrate that in CPTs and glioblastoma, wild-type $X$. tropicalis p53 is able to limit tumor progression. Namely, tp53 nullizygous choroid plexus tumors and gliomas demonstrate more malignant histological characteristics, higher proliferation and increased chromosomal instability, in line with the published clinical human and mice experimental data ${ }^{3,9,65}$.

390 In this paper we also describe the first tp53 nullizygous $X$. tropicalis line and demonstrate that 391 Xenopus tp53 behaves, analogous to TP53 in Li-Fraumeni, as a tumor suppressor gene for syndromic 392 Xenopus cancer development. We demonstrate that tp53 mutations are predisposing for a Li-Fraumeni 
tumor spectrum, namely hematological malignancies and sarcomas, admittingly with a long latency of $>1$ year. We believe the latter to be attributable to the life-span of $X$. tropicalis that is substantially longer $(>3 x)$ than mice and may also underlie interspecies physiological differences. Interestingly, our $t p 53^{\Delta 4 \mathrm{var} / \Delta 4 \mathrm{var} 2}$ compound heterozygote line enables easy identification of each $t p 53$ allele. This allows tracking of local chromosomal instability during cancer initiation and progression by straightforward amplicon sequencing of both distinct tp53 CRISPR/Cas9 deletion scars, permitting rapid assessment of tp53 focal amplification, aneuploidy events or loss of heterozygosity.

When comparing our novel $X$. tropicalis cancer models to established zebrafish models, neither tp53 nor rb1 mutant zebrafish have been reported to develop Li-Fraumeni-related hematological malignancies, SC-PaNEC or CPT ${ }^{27}$. In fact, in contrast to the clinical situation, tp53 mutant fish develop predominantly malignant peripheral nerve sheath tumors and rb1 TALENs F0 edited $t p 53$ mutant fish develop primitive neuro-ectodermal tumors and medulloblastoma ${ }^{66,67}$.

In the comparison to established mammalian cancer models, we believe that Xenopus holds unique experimental advantages such as extremely straightforward tissue-restrictive CRISPR/Cas9 delivery and multiplexing in externally developing embryos ${ }^{18}$. Additionally, the $X$. tropicalis SC-PaNEC, glioblastoma and choroid plexus cancer models we report here are competitive with or outperform the existing Rb1/Tp53-inactivated mice models in terms of latency and penetrance of tumor development (Table S4).

In synopsis, we demonstrate the use of tissue-restricted multiplexed inactivation in Xenopus embryos to functionally identify novel tumor suppressor combinations in vivo. This nicely complements AAVmediated in vivo murine CRISPR screens ${ }^{14,45}$. We established novel highly penetrant and short latency genetic $X$. tropicalis cancer models upon multiplex in vivo CRISPR/Cas9-mediated inactivation of the $r b 1$, rbl1 and tp53 tumor suppressor genes. These models will generate novel opportunities for gene function interrogation, therapeutic target identification and pre-clinical drug studies. Finally, showcasing the possibilities of multiplexed CRISPR/Cas9 in delineating novel driver mutations in cancer, we establish

417 formal proof of rb/1 as a tumor suppressor in glioblastoma and small-cell pancreatic neuroendocrine 418 carcinoma.

\section{Material and Methods}

\section{Generation of $X$.tropicalis mosaic mutants by CRISPR/Cas9}

422 All gRNAs were designed with the CRISPRScan algorithm (http://www.crisprscan.org/) ${ }^{68}$. In this study 423 following sequences were targeted. Rb1 5'-GCTGTATGATTGTGCTGTACCGG-3', $r b / 1$ 
TGGGCTTGCGCGCTGATGTGGGG, $\quad$ tp53 $3^{\text {cr } 1}$

5'-CCTCAACTGAGGATTACGCAGGG-3',

$\operatorname{tp} 53^{\mathrm{cr} 2} \quad 5^{\prime}-$ GAAGAGCTTGTTGAGGTCGGTGG-3', pten $^{\text {cr1 }}$

5'-GCGCTTGGGACCTGCTGTTGAGG-3', pten ${ }^{\text {cr2 }} 5^{\prime}-$

426 GAGTTACAATTCCCAGCCAAAGG-3'. gRNAs were generated by in vitro transcription, employing the oligos

427 shown in supplementary table $5 \mathrm{~A}$, and quality control and quantification was performed as described

428 before ${ }^{69,70}$. Recombinant NLS-Cas9-NLS protein was generated as previously described ${ }^{14}$. Natural $X$.

429 tropicalis matings were performed and embryos were microinjected in either the two- or the eight-cell

430 stage with injection mixes containing precomplexed gRNA and Cas9 protein as shown in supplementary

431 table 5B. Approval was obtained from the Ethical Committee for Animal Experimentation, Ghent

432 University, Faculty of Science and VIB-Site Ghent (EC2018-079). All methods were carried out in

433 accordance with the relevant guidelines set out by this committee.

\section{Next-generation amplicon sequencing}

435 Targeted amplicon sequencing followed by BATCH-GE analysis was performed for quantitative 436 assessment of genome editing, with the primer pairs shown in supplementary table $5 \mathrm{C}$, as described 437 before ${ }^{71-73}$. For tp53 genotyping of animals after necropsy, spinal cord (ectoderm targeted) or heart 438 (anterior endoderm targeted) tissue were lysed overnight in lysis buffer (50 mM Tris pH 8.8, 1 mM EDTA, $4390.5 \%$ Tween-20, $200 \mu \mathrm{g} / \mathrm{ml}$ proteinase $\mathrm{K}$ ) and targeted amplicon sequencing of tp53 was performed 440 identically as above. For next-generation amplicon sequencing from laser-capture microdissected 441 tumors, PCR input DNA was generated as previously described ${ }^{69}$. PCR amplification relevant genomic 442 regions was performed with the (nested) primers shown in table 5D. All quantitative genome editing 443 efficiencies and INDEL variants, with their relative frequencies, in this study are shown in supplementary 444 Table 1.

\section{Ex-vivo micro-CT imaging}

446 For ex-vivo micro-CT imaging of tp53 heterozygous animals, cone-beam micro-CT was employed 447 (Triumph-II, TriFoil Imaging, Northridge, CA, USA). A high-resolution micro-CT imaging protocol was used 448 with the following acquisition parameters: one bed position (90 mm field of view), circular trajectory 449 with continuous rotation over 360 degrees, $50 \mu \mathrm{m}$ focal spot size, $50 \mu \mathrm{m}$ detector pixel size, 1.3 times 450 magnification, 512 projections, $370 \mu \mathrm{A}$ tube current and $50 \mathrm{kVp}$ tube voltage, resulting in a total 451 acquisition time of 14 minutes. The acquired projection data were reconstructed into a three-

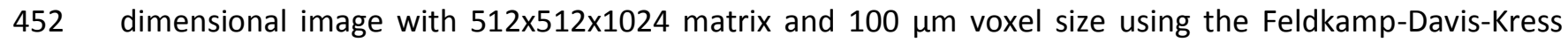
453 algorithm. 


\section{Flow Cytometry and Natt-Herrick leukocyte counting}

455

456

457

458

459

460

Peripheral blood was collected from wild-type or mutant $X$. tropicalis by toe clipping and immediately suspended in cold $0.66 x$ PBS (amphibian PBS or APBS). Cells were fixed for 20 minutes using fresh $2 \%$ paraformaldehyde, blocked/permeabilized in 2\% Natural Goat Serum, 1\% BSA (VWR) and 0.1\% Tween 20 (Sigma-Aldrich) in APBS solution. After 10 minutes of blocking and permeabilization, cells were incubated for one hour either with a 1:5 dilution of Rat anti Human CD3:Alexa Fluor ${ }^{\circledR} 647$ (0.05 mg/mL) (clone CD312; Bio-Rad) or with undiluted CD8 (AM22) mouse hybridoma supernatant $(0.05 \mathrm{mg} / \mathrm{mL})$. Cells incubated with hybridoma supernatant were additionally incubated with DyLight ${ }^{\circledR} 488$ secondary antibody for one hour. Finally, cells were acquired on a 5 laser-BD LSR Fortessa flow cytometer (BD Biosciences) and analyzed using the FlowJo software (Tree Star). For validation, blood was collected by cardiac bleed from euthanized animals and diluted 1:50 in Natt-Herrick (NH) staining solution, prepared as described before ${ }^{74}$. NH preperates were loaded in a Buerker hemocytometer (Marienfeld) where after leukocytes and red blood cells (RBC) were counted. For each data-point in the statistical analysis, leukocytes and RBC were counted in twelve $0.04 \mathrm{~mm}^{2}$ regions. Percentage leukocytes was calculated as (\#leukocytes/(\#leukocytes+\#RBC)).

\section{Histology, immunohistochemistry and nuclear modeling of glioblastoma cells}

All macroscopic pictures were taken with a Carl Zeiss StereoLUMAR.V12 stereomicroscope. For quantification of SC-PaNEC sizes, ImageJ was used. For histology, tissues were fixed overnight in $4 \%$ paraformaldehyde (PFA) at $4^{\circ} \mathrm{C}$. Bone-containing tissues (e.g. cranial structures) were decalcified by Morse's solution ( $10 \%$ sodium citrate and $22.5 \%$ formic acid) for 6 hours at room temperature. All tissue samples were dehydrated, imbedded in paraffin and $5 \mu \mathrm{M}$ tissue sections were made by microtomy. For classical histopathology, slides were stained with hematoxylin and eosin. Choroid plexuses were analyzed by a clinical pathologist (D. C.) and assigned a WHO grade. Immunohistochemistry was performed as previously described with primary antibodies: anti-PCNA antibody (PC10; Dako), anti-pHH3 antibody (IHC-00061; Bethyl laboratories), anti-CD3 antibody (clone CD3-12; Bio-Rad), anti-GFAP (Z0334; DAKO), anti-cleaved-caspase3 (9661; CST) and anti-EZH2 antibody (A304-197A-T; Bethyl Laboratories) ${ }^{75}$. TUNEL was performed using the In Situ Cell Death Detection Kit, AP (11684809910, Roche) according to manufacturer's instructions. In all immunohistochemical experiments, omission of either the primary antibody or the TUNEL labeling mix served as a negative control. For immunofluorescent (IF) goat antimouse DyLight-633 (ThermoFisher) and goat anti-rabbit DyLight-633 (ThermoFisher) was used. All IF and TUNEL samples where counterstained with Hoechst-33342. SC-PaNEC PCNA IF was captured with a Leica 
TCS LSI zoom confocal microscope. Choroid plexus tumor PCNA IF and Hoechst-33324 for glioma nuclear modeling were collected on an LSM880 Airyscan (Carl Zeiss, Jena, Germany) using either a LD LCI PlanApochromat 25x/0.8 Imm Korr DIC M27 or a 63x PlanApo NA:1.4 oil immersion DIC M27 objective. The operating software was ZEN blue 2.3. The Airyscan detector was used in both the fast and full superresolution mode. A pixel reassignment algorithm in combination with a Wiener filter were carried out post acquisition. In order to gain nuclear 3D information, z-stacks were recorded and a 3D reconstruction of relevant z-stacks was made in Volocity 6.3.0 (Perking Elmer). Furthermore, nuclear material from glioblastoma cells was segmented, employing MIB, and a 3D model of the nuclear membrane was build using Imaris (Bitplane) ${ }^{76}$. TUNEL, pHH3 and GFAP IF was captured on a Leica TCS SP5 confocal microscope using the tile scan and image stitching function. For IHC with DAB detection of EZH2, PCNA, CD3 and Cleaved-Caspase 3, Goat Anti-Rabbit IgG (H+L) (VEC.BA-1000; Vector laboratories) was used. Signal was developed using the VECTASTAIN Elite ABC HRP Kit (PK-6100; Vector laboratories) and ImmPACT DAB Peroxidase (SK-4105; Vector laboratories). Samples were counterstained 1 minute with hematoxylin. Imaging of H\&E stained and DAB/hematoxylin stained sections was performed with a Zeiss Axio Scan.Z1 equipped with a 20X Plan-Apochromat 0.8 NA dry objective, using a Hitachi HVF202SCL camera. High-magnification photomicrographs of glioblastoma cells were taken with an Zeiss Axioscope using a 100x oil objective. To quantity cell proliferation in tumors, we performed automated counting of $\mathrm{pHH}^{+}$cells using ImageJ, normalizing $\mathrm{pHH}^{+}$cells over tumor section volume.

\section{Acknowledgments}

Research in the authors' laboratory is supported by the Research Foundation - Flanders (FWOVlaanderen) (grants G0A1515N and G029413N), by the Belgian Science Policy (Interuniversity Attraction Poles - IAP7/07) and by the Concerted Research Actions from Ghent University (BOF15/GOA/011). Further support was obtained by the Hercules Foundation, Flanders (grant AUGE/11/14) and the Desmoid Tumor Research Foundation. T.N. is funded by "Kom op tegen Kanker" (Stand up to Cancer), the Flemish cancer society and previously held PhD fellowship with VLAIO-HERMES during the course of this work. We thank the Xenopus laevis Resource for Immunobiology (Rochester, NY, NIH R24 AI059830) 511 for the kind gift of monoclonal antibody AM20 (10A91, CD8). We are indebted to Tim Deceuninck for 512 animal care, Kelly Lemeire for technical assistance with TUNEL staining and Marjolein Carron for BATCH513 GE analysis of sequencing data. We would like to thank the VIB Biolmaging Core, and in particular Chris 514 Guerin, Eef Parthoens and Anneke Kremer, for access to the instrument park, training and support. In 

manuscript.

\section{Author information}

\section{Affiliations}

Department of Biomedical Molecular Biology, Ghent University, Ghent, Belgium Noelanders, Kris Vleminckx

524 Thomas Naert, Dionysia Dimitrakopoulou, Dieter Tulkens, Christian Vanhove, Jo Van Dorpe,

525 David Creytens, Kris Vleminckx

526 Center for Medical Genetics, Ghent University, Ghent, Belgium

527 Kris Vleminckx

VIB Center for Inflammation Research, Ghent, Belgium

529 Gert Van Isterdael

530 Laboratory for Pharmaceutical Biotechnology, Ghent University, B-9000 Ghent, Belgium.

531 Dieter Deforce

Department of Pathology, Ghent University and Ghent University Hospital, Ghent, Belgium

534 David Creytens, Jo Van Dorpe

Infinity lab, Ghent University Hospital, Ghent, Belgium.

536 Christian Vanhove

\section{Contributions}

539 T.N, D.Di. and K.V. designed the study. D.Di., T.N, D.T. and R.N. were involved in generation and 540 phenotyping of the tp53 mutant $X$. tropicalis. T.N. performed genome engineering and phenotyping of all 541 retinoblastoma and retinoblastoma/tp53 mutants. T.N., L.E. and D.De were involved in laser-capture 542 microdissection and downstream analysis. D.C. and J.v.D performed pathological analysis. D.T., D.Di. and 543 G.v.I performed flow cytometry experiments. T.N. performed cancer genomic database mining. C.V. 544 performed X-ray imaging. S.D. provided technical assistance throughout the project. T.N. and K.V. wrote 545 the manuscript. 


\section{References}

548 1. Konukiewitz, B. et al. Pancreatic neuroendocrine carcinomas reveal a closer relationship to ductal 549 adenocarcinomas than to neuroendocrine tumors G3. Hum. Pathol. 77, 70-79 (2018).

550 2. Glenn, S. T. et al. Conditional deletion of p53 and Rb in the renin-expressing compartment of the 551 pancreas leads to a highly penetrant metastatic pancreatic neuroendocrine carcinoma. Oncogene 33, 5706-15 (2014).

3. Tong, Y. et al. Cross-Species Genomics Identifies TAF12, NFYC, and RAD54L as Choroid Plexus

4. McLendon, R. et al. Comprehensive genomic characterization defines human glioblastoma genes

5. Chow, L. M. L. et al. Cooperativity within and among Pten, p53, and Rb pathways induces high-

6. George, J. et al. Comprehensive genomic profiles of small cell lung cancer. Nature $524,47-53$ (2015).

7. Hann, C. L. \& Rudin, C. M. Management of small-cell lung cancer: incremental changes but hope

8. Basturk, O. et al. The High-grade (WHO G3) Pancreatic Neuroendocrine Tumor Category Is

10. Chow, R. D. et al. AAV-mediated direct in vivo CRISPR screen identifies functional suppressors in

12. Costa, C., Paramio, J. M. \& Santos, M. Skin Tumors Rb(eing) Uncovered. Front. Oncol. 3, 307

11. Dannenberg, J.-H., Schuijff, L., Dekker, M., Van Der Valk, M. \& Te Riele, H. Tissue-specific tumor suppressor activity of retinoblastoma gene homologs p107 and p130. (2004).

13. Robanus-Maandag, E. et al. p107 is a suppressor of retinoblastoma development in pRb-deficient (2013). progression and N-myc gene amplification. EMBO J. 26, 784-94 (2007).

581 16. Xiao, A., Wu, H., Pandolfi, P. P., Louis, D. N. \& Van Dyke, T. Astrocyte inactivation of the pRb 
pathway predisposes mice to malignant astrocytoma development that is accelerated by PTEN mutation. Cancer Cell 1, 157-68 (2002).

17. Lu, X. et al. Selective Inactivation of p53 Facilitates Mouse Epithelial Tumor Progression without Chromosomal Instability. Mol. Cell. Biol. 21, 6017-6030 (2001).

18. Naert, T., Van Nieuwenhuysen, T. \& Vleminckx, K. TALENs and CRISPR/Cas9 fuel genetically

19. DeLay, B. D. et al. Tissue-specific Gene Inactivation in Xenopus laevis : Knockout of Ihx1 in the engineered clinically relevant Xenopus tropicalis tumor models. genesis 55, e23005 (2017). Kidney with CRISPR/Cas9. Genetics genetics.300468.2017 (2017). doi:10.1534/genetics.117.300468

20. Wirt, S. E. \& Sage, J. p107 in the public eye: an Rb understudy and more. Cell Div. 5, 9 (2010).

21. Li, F. P. \& Fraumeni, J. F. Soft-tissue sarcomas, breast cancer, and other neoplasms. A familial syndrome? Ann. Intern. Med. 71, 747-52 (1969).

22. Malkin, D. et al. Germ line p53 mutations in a familial syndrome of breast cancer, sarcomas, and other neoplasms. Science 250, 1233-8 (1990).

23. Bougeard, G. et al. Revisiting Li-Fraumeni Syndrome From TP53 Mutation Carriers. J. Clin. Oncol. 33, 2345-2352 (2015).

24. Donehower, L. A. et al. Mice deficient for p53 are developmentally normal but susceptible to spontaneous tumours. Nature 356, 215-221 (1992).

25. Jacks, T. et al. Tumor spectrum analysis in p53-mutant mice. Curr. Biol. 4, 1-7 (1994).

26. Purdie, C. A. et al. Tumour incidence, spectrum and ploidy in mice with a large deletion in the p53

27. Ignatius, M. S. et al. tp53 deficiency causes a wide tumor spectrum and increases embryonal

28. Berghmans, S. et al. tp53 mutant zebrafish develop malignant peripheral nerve sheath tumors.

29. Manning, A. L., Benes, C. \& Dyson, N. J. Whole chromosome instability resulting from the

30. Levine, A. J. p53, the Cellular Gatekeeper for Growth and Division. Cell 88, 323-331 (1997).

31. Eischen, C. M. Genome Stability Requires p53. Cold Spring Harb. Perspect. Med. 6, a026096 (2016).

32. Naert, T. \& Vleminckx, K. CRISPR/Cas9 disease models in zebrafish and Xenopus: The genetic

33. Lau, C.-H. \& Suh, Y. In vivo genome editing in animals using AAV-CRISPR system: applications to 
assessment. Sci. Rep. 6, 30330 (2016).

35. Neely, H. R., Guo, J., Flowers, E. M., Criscitiello, M. F. \& Flajnik, M. F. 'Double-duty' conventional dendritic cells in the amphibian Xenopus as the prototype for antigen presentation to B cells. Eur. J. Immunol. 48, 430-440 (2018).

36. Ward, J. M. et al. Splenic marginal zone B-cell and thymic T-cell lymphomas in p53-deficient mice. Lab. Invest. 79, 3-14 (1999).

37. Wang, Z. et al. Pseudo-hemorrhagic region formation in pancreatic neuroendocrine tumors is a result of blood vessel dilation followed by endothelial cell detachment. Oncol. Lett. 15, 42554261 (2018).

38. Montoliu, L. Mendel: a simple excel workbook to compare the observed and expected distributions of genotypes/phenotypes in transgenic and knockout mouse crosses involving up to three unlinked loci by means of a $\chi 2$ test. Transgenic Res. 21, 677-681 (2012).

39. Chiou, S.-H. et al. Pancreatic cancer modeling using retrograde viral vector delivery and in vivo CRISPR/Cas9-mediated somatic genome editing. Genes Dev. 29, 1576-85 (2015).

40. Maresch, R. et al. Multiplexed pancreatic genome engineering and cancer induction by transfection-based CRISPR/Cas9 delivery in mice. Nat. Commun. 7, (2016).

41. van Zoest, I. D., Heijmen, P. S., Cruijsen, P. M. J. M. \& Jenks, B. G. Dynamics of background adaptation in Xenopus laevis: Role of catecholamines and melanophore-stimulating hormone. Gen. Comp. Endocrinol. 76, 19-28 (1989).

42. Marino, S., Vooijs, M., van Der Gulden, H., Jonkers, J. \& Berns, A. Induction of medulloblastomas in p53-null mutant mice by somatic inactivation of $\mathrm{Rb}$ in the external granular layer cells of the cerebellum. Genes Dev. 14, 994-1004 (2000).

43. McEvoy, J. et al. Coexpression of normally incompatible developmental pathways in retinoblastoma genesis. Cancer Cell 20, 260-75 (2011).

44. Louis, D. N. et al. The 2016 World Health Organization Classification of Tumors of the Central Nervous System: a summary. Acta Neuropathol. 131, 803-820 (2016).

45. Chow, R. D. et al. AAV-mediated direct in vivo CRISPR screen identifies functional suppressors in glioblastoma. Nat. Neurosci. 20, 1329-1341 (2017).

46. Jin, X. et al. Targeting glioma stem cells through combined BMI1 and EZH2 inhibition. Nat. Med. 23, 1352-1361 (2017).

47. Steed, T. C. et al. Differential localization of glioblastoma subtype: implications on glioblastoma pathogenesis. Oncotarget 7, 24899-24907 (2016).

48. Koul, D. PTEN signaling pathways in glioblastoma. Cancer Biol. Ther. 7, 1321-5 (2008).

49. Schaffer, B. E. et al. Loss of p130 accelerates tumor development in a mouse model for human small-cell lung carcinoma. Cancer Res. 70, 3877-83 (2010).

50. Yachida, S. et al. Small Cell and Large Cell Neuroendocrine Carcinomas of the Pancreas are 

J. Surg. Pathol. 36, 173-184 (2012).

51. Abou-El-Ardat, K. et al. Comprehensive molecular characterization of multifocal glioblastoma proves its monoclonal origin and reveals novel insights into clonal evolution and heterogeneity of glioblastomas. Neuro. Oncol. 19, 546-557 (2017).

52. Wiedemeyer, W. R. et al. Pattern of retinoblastoma pathway inactivation dictates response to

53. Cerami, E. et al. The cBio Cancer Genomics Portal: An Open Platform for Exploring

54. Gao, J. et al. Integrative Analysis of Complex Cancer Genomics and Clinical Profiles Using the

55. Liu, F. et al. MicroRNA-106b-5p boosts glioma tumorigensis by targeting multiple tumor

59. Vanderluit, J. L. et al. The Retinoblastoma family member $\mathrm{p} 107$ regulates the rate of progenitor

60. Harb, G., Vasavada, R. C., Cobrinik, D. \& Stewart, A. F. The retinoblastoma protein and its

56. Vitucci, M. et al. Genomic profiles of low-grade murine gliomas evolve during progression to glioblastoma. Neuro. Oncol. 19, 1237-1247 (2017).

57. Wojton, J. et al. Systemic Delivery of SapC-DOPS Has Antiangiogenic and Antitumor Effects Against Glioblastoma. Mol. Ther. 21, 1517-1525 (2013).

58. Vanderluit, J. L. et al. p107 regulates neural precursor cells in the mammalian brain. J. Cell Biol. 166, 853-863 (2004).

\section{Cai, E. P. et al. Rb and p107 are required for alpha cell survival, beta cell cycle control and} glucagon-like peptide-1 action. Diabetologia 57, 2555-2565 (2014).

62. Vasavada, R. C., Cozar-Castellano, I., Sipula, D. \& Stewart, A. F. Tissue-specific deletion of the retinoblastoma protein in the pancreatic beta-cell has limited effects on beta-cell replication, mass, and function. Diabetes 56, 57-64 (2007).

63. Konukiewitz, B. et al. Somatostatin receptor expression related to TP53 and RB1 alterations in pancreatic and extrapancreatic neuroendocrine neoplasms with a Ki67-index above 20\%. Mod. Pathol. 30, 587-598 (2017).

64. Konukiewitz, B. et al. Pancreatic neuroendocrine carcinomas reveal a closer relationship to ductal adenocarcinomas than to neuroendocrine tumors G3. Hum. Pathol. 77, 70-79 (2018).

65. Merino, D. M. et al. Molecular Characterization of Choroid Plexus Tumors Reveals Novel Clinically 
zebrafish by TALEN-mediated somatic inactivation of the retinoblastoma1 tumor suppressor rb1. Sci. Rep. 5, 13745 (2015).

67. Shim, J. et al. Development of zebrafish medulloblastoma-like PNET model by TALEN-mediated somatic gene inactivation. Oncotarget 8, 55280-55297 (2017).

68. Moreno-Mateos, M. A. et al. CRISPRscan: designing highly efficient sgRNAs for CRISPR-Cas9 targeting in vivo. Nat. Methods 12, 982-8 (2015).

69. Naert, T. \& Vleminckx, K. CRISPR/cas9-mediated knockout of RB1 in xenopus tropicalis. Methods in

70. Naert, T. \& Vleminckx, K. Methods for CRISPR/Cas9 Xenopus tropicalis Tissue-Specific Multiplex

71. Steyaert, W., Boel, A., Coucke, P. \& Willaert, A. BATCH-GE: Analysis of NGS Data for Genome Genome Engineering. Methods Mol. Biol. 1865, 33-54 (2018).

72. Naert, T. \& Vleminckx, K. Genotyping of CRISPR/Cas9 Genome Edited Xenopus tropicalis. Methods Editing Assessment. Methods Mol. Biol. 1865, 83-90 (2018). Mol. Biol. 1865, 67-82 (2018).

73. Szenker-Ravi, E. et al. RSPO2 inhibition of RNF43 and ZNRF3 governs limb development independently of LGR4/5/6. Nature 557, 564-569 (2018).

74. Maxham, L. A., Forzán, M. J., Hogan, N. S., Vanderstichel, R. V. \& Gilroy, C. V. Hematologic reference intervals for Xenopus tropicalis with partial use of automatic counting methods and reliability of long-term stored samples. Vet. Clin. Pathol. 45, 291-299 (2016).

75. Naert, T. \& Vleminckx, K. Cancer Models in Xenopus tropicalis by CRISPR/Cas9 Mediated Knockout of Tumor Suppressors. Methods Mol. Biol. 1865, 147-161 (2018).

76. Belevich, I., Joensuu, M., Kumar, D., Vihinen, H. \& Jokitalo, E. Microscopy Image Browser: A Platform for Segmentation and Analysis of Multidimensional Datasets. PLOS Biol. 14, e1002340 (2016). 


\section{Figure Legends}

718 Figure 1. Tp53 mutant $X$. tropicalis develop hematological malignancy and sarcomas. (A) Kaplan-Meier 719 survival analysis on cohorts consisting of two clutches of $t p 53$ homozygous knockout ( $n=6$; red and $n=7$; 720 blue) and one clutch of heterozygous tp53 knockout ( $n=14$; black) $X$. tropicalis. Statistical analysis was 721 done using the Prism Mantel-Cox test (ns - not significant; ${ }^{* *} p<0.01 ; * * * p<0.001$ ). Chi-Squared 722 values and the Hazard ratios are listed in Supplementary table 2A. (B) X-ray imaging of a 28-month old $723 t p 53^{+/ \Delta 4 v a r 2}$ demonstrating ectopic calcified structures (white arrows). (C) (Left panels) In the wild-type 724 spleen, $\mathrm{CD}^{+}$T-cells form a ring-like structure around the PCNA ${ }^{+}$B-cells located in the white pulp. (Right 725 panels) Disruption of a normal $\mathrm{CD}^{+}$ring-like structure observed in a $t p 53^{\Delta 4 \mathrm{var} 2 / \Delta 4 \mathrm{var} 2}$ animal is indicative 726 of hematological malignancy. (D) Pie chart summarizing the observed splenic immunostaining for CD3 727 and PCNA in $t p 53^{\Delta 4 v a r 1 / \triangle 4 v a r 2}$ animals. Staining patterns being either normal or with loss of the typical CD3 ${ }^{+}$ 728 ring structure with or without ectopic staining of PCNA in the red pulp. (E) Photomicrograph of Natt729 Herrick stained blood demonstrating a cluster of leukocytes and a normal nucleated erythrocyte. Inset 730 demonstrates a high magnification photomicrograph of a lymphoblast. (F) Flow cytometry analysis 731 reveals an increased number of both $\mathrm{CD}^{+}$(left) and $\mathrm{CD} 8^{+}$lymphoblasts (right) in peripheral blood of a $732 t p 53^{\Delta 4 \mathrm{var} 1 / \Delta 4 \mathrm{var} 2}$ animal, when compared to an age-matched control. (G) Histopathology of the liver reveals 733 diffuse infiltration of T-lymphoblasts, also enriched within liver capillary (white arrow; top inset) and in 734 between the liver parenchymal cells (black arrow). (H) Sarcoma in a $t p 53^{+/ \Delta 4 v a r 2}$ animal observed upon 735 gross examination, with associated histopathology and demonstration of malignant nature by PCNA 736 proliferation staining. White scale bar is $100 \mu \mathrm{m}$ and black scale bar is $5 \mu \mathrm{M}$.

737 Figure 2. Small-cell pancreatic neuroendocrine carcinoma (SC-PaNEC) in $X$. tropicalis upon mosaic 738 CRISPR/Cas9 genome editing of $r b 1$ and $r b / 1$ in the anterior endoderm. (A) Breeding and CRISPR/Cas9 739 injection scheme demonstrating the generation of the single mutant KO (smKO -orange) (rb1 $740 \mathrm{CRISPR} /$ Cas9 injection in embryos obtained from a $t p 53^{\Delta 4 \mathrm{var} 1 /+} \times \operatorname{tp} 53^{\Delta 4 \mathrm{var} 2 /+}$ intercross), double mutant KO 741 (dmKO - blue) (rb1/rb/1 CRISPR/Cas9 injection in embryos obtained from a $t p 53^{\Delta 4 v a r 1 /+} \times \operatorname{tp} 53^{\Delta 4 v a r 2 /+}$ 742 intercross) and triple mutant KO (tmKO - purple) (rb1/rb/1/tp53 CRISPR/Cas9 injection in embryos 743 obtained from intercrossing WT animals). (B) Typical external pathology of a pancreatic tumor occurring 744 in a tmKO animal. The inset shows an age-matched control pancreas. (C) Tumor incidence curves and 745 Kaplan-Meier analysis comparing smKO $(n=13), \operatorname{dmKO}(n=29)$ and tmKO $(n=22)$ genotypes. Statistical 746 analysis, done using the Prism Mantel-Cox test, revealed that SC-PaNEC incidences were significantly 747 different across the experimental setups $(p<0.0001)$. Chi-Squared values and the Hazard ratios are listed 
748 in supplementary table 2C. (D) H\&E stain of pancreatic tumors shows recurrent histological features of 749 SC-PaNEC with necrotic foci (nec). The black arrow indicates non-neoplastic pancreatic tissue. The inset 750 shows, under higher magnification, diffuse sheets of poorly differentiated cells with small blue round 751 morphology, nuclear pleomorphism and hemorrhages (yellow arrows). (E) SC-PaNEC sections 752 immunostained for $\mathrm{pHH} 3$ or PCNA, counterstained with Hoechst-33342, reveal high proliferative 753 capacity. (F) Laser-capture microdissection (LCM) of dmKO SC-PaNEC reveals chromosomal instability in $754 t p 53^{-/}$tumors. In $t p 53^{+/ d e l 4}$ tumors the allelic ratio of the mutant allele remains around $50 \%$ (right graph). 755 In contrast, two out of three tp53\% tumors show substantial deviation from the expected allelic ratios 756 (50\% each variant) (left graph). (G) Percentage of mutant $r b / 1$ and tp53 reads observed in LCM-derived 757 SC-PaNECs sampled from either tmKO (left) or dmKO (right) animals. Red bullets and blue bullets 758 demarcate SC-PaNECs with monoallelic inactivating tp53 mutations in the tmKO and dmKO setup, 759 respectively. White scale bar is $500 \mu \mathrm{m}$, black scale bar is $50 \mu \mathrm{m}$.

760 Figure 3. $\boldsymbol{R b} \mathbf{1}$ and $\boldsymbol{r b} / \mathbf{1}$ crispants (ectodermal targeted) develop retinoblastoma, excessive black skin 761 pigmentation and a spectrum of brain tumors. (A) CRISPR/Cas9 targeting of $r b 1$ and $r b / 1$ in ectodermal 762 lineage via unilateral injection of an animal-dorsal blastomere. (B) Unilateral ectodermal targeting of $763 \mathrm{rb1} / \mathrm{rb} / 1 \mathrm{CRISPR} / \mathrm{Cas9}$, in tp53 wild-type, heterozygous or compound heterozygous background, results in 764 crispants developing externally visible retinoblastoma $(75 \% ; n=16)$ and excessive skin pigmentation 765 (44\%; $n=16)$. Inset shows an animal from the same clutch without external symptoms. (C) H\&E stained 766 section illustrating increased pigment deposition internal to the stratified epithelium (black arrow). Inset 767 is an animal from the same clutch showing normal pigment deposition. (D) H\&E stained section of the 768 animal shown in panel A. Unilateral retinoblastoma (black arrow) can clearly be distinguished. Inset 769 shows higher magnification of the retinoblastoma illustrating the classic histopathological characteristics 770 (homer-wright rosettes). (E) H\&E stained horizontal brain section (anterior side of the animal to the left) 771 revealing multiple neoplasms. Two distinct poorly differentiated, highly malignant, small-blue round cell 772 tumors of unknown origin are shown, most likely representing trilateral retinoblastoma and 773 medulloblastoma (white arrow). Furthermore, a choroid plexus neoplasm can clearly be distinguished 774 within an expanded ventricle (black arrow) and the forebrain harbors a glioblastoma lesion (red arrow). 775 Inset shows the normal $X$. tropicalis brain architecture. White scale bar is $1 \mathrm{~mm}$, black scale bar is 100 $776 \mu \mathrm{M}$.

777 Figure 4: Choroid plexus (CP) tumors arising in $r b 1 /$ rbl1 crispants differ in grade according to their tp53 778 genotype. (A) H\&E stained sections of choroid plexuses representative for the CPT tumor grades 
779

780

781

782

783

784

785

observed within the clutch. (Top panels) Normal choroid plexus architecture consists out of a layer of ependymal cells, underlying capillaries and pia mater. Insets demonstrate both gross (left) and histological (right) normal CP architecture. Note that in Xenopus, the erythrocytes are nucleated. (Middle panels) WHO grade 1 (G1) CP neoplasm with cellular disarray, (pseudo)-stratification, atypia and loss of polarization (black arrow). (Bottom panels) WHO > G1 CP neoplasm with pronounced atypia and aggressive growth characteristics. (B) Choroid plexus tumor in a $t p 53^{+/-}$animal showcasing clear continuity between normal CP and neoplastic tissue. (C) Association between tp53 genotype and grade of choroid plexus lesions ( $p<0.01$; Table S2H). (D) > G1 CP neoplasm immunostained for PCNA (red), counterstained with Hoechst-33342, reveals high proliferative capacity within highly atypical tumor areas (white arrow). (E) Laser-capture microdissection (LCM) of CP tumors (CPT) reveals chromosomal instability, as can be appreciated by the differential tp53 allelic ratios between control and CPT tissues. Left $\left(t p 53^{\Delta 4 / \Delta 4}\right)$ control bar show the average of LCM control tissues $(n=2)$. Right $\left(t p 53^{\Delta 4 /+}\right)$ control is the allelic ratio in spinal cord control tissue from the animal carrying CPT-5. Gray scale bar is $500 \mu \mathrm{M}$, white scale bar is $100 \mu \mathrm{M}$, black scale bar is $50 \mu \mathrm{M}$. White scale bar in panel $\mathrm{D}$ is $10 \mu \mathrm{M}$.

Figure 5: $R \boldsymbol{b} / 1$ functions as a tumor suppressor in glioblastoma, while tp53 inactivation underlies progression. (A) H\&E stained sections of Xenopus forebrains containing representative histopathology of low-grade glioma (left-middle) and high-grade glioblastoma (right) occurring across different experimental conditions. (B) (Left panel) Low grade glioma with the occasional multinucleated giant cell in tp53 wild-type and heterozygous animals, targeted in the ectodermal lineage with $r b 1$ and $r b / 1$ CRISPR/Cas9. (Right panel) However, when similarly targeting $r b 1$ and $r b / 1$ in a tp53 nullizygous animals, higher-grade glioblastoma lesions develop. (C) Quadruple multiplex CRISPR/Cas9-mediated targeting of $r b 1, r b / 1, t p 53$ and pten in the ectodermal lineage leads to fully penetrant $(100 \% ; n=13)$ development of high-grade glioblastoma lesions at day 42. (D) Bar plot representing absence or presence of either lowgrade glioma or high-grade glioblastoma lesions across the performed experimental setups. Genes shown in bold were edited in the ectodermal lineage by CRISPR/Cas9, while $t p 53$ genotypes, shown in grey, were germline inherited. (E) (top) PCNA immunostaining reveals high proliferative index in glioblastoma cells. (bottom) EZH2 immunostaining reveals EZH2 expression in glioblastoma cells, but absence of expression in the subventricular zone (black arrow). Black scale bar is $100 \mu \mathrm{M}$ and white scale bar is $20 \mu \mathrm{M}$.

Supplemental movie 1: Central glioblastoma cell with nuclear aneuploidy and increased number of nucleoli, surrounded by normal neurons demonstrating two nucleoli. 3D-reconstruction of Hoechst- 
81033342 stained nuclei followed by segmented 3D-model of glioblastoma nuclear membrane. This movie

811 demonstrates heavily increased number of nucleoli within the glioblastoma cell.

812 Supplemental movie 2: Massive interconnected nuclear abnormalities in glioblastoma. 3D-

813 reconstruction of Hoechst-33342 stained nuclei followed by segmented 3D-model of glioblastoma

814 nuclear membrane. This movie demonstrates that nuclear material in seemingly disconnected

815 glioblastoma cells can in fact be interconnected.

\section{Supplementary data: Tables}

817 Table S1: Genotyping by PCR amplification, sequencing (MiSeq) and BATCH-GE analysis. Genotyping 818 performed throughout this study after BATCH-GE processing with genome editing efficiencies and 819 variant calls, subdivided in letter-coded sheets.

820 Table S2: Statistical Analyses. Raw data and statistical tests performed throughout this study, subdivided 821 in letter coded sheets.

822 Table S3: Analysis of co-occurrence of RB1 and RBL1 alterations in human glioma.

823 Table S4: Comparison between established, closest prior art, mice cancer models and the novel 824 Xenopus models presented in this study in terms of time-to-onset and penetrance of cancer 825 phenotypes.

826 Table S5: Primer sequences used for PCR amplification, oligo sequences used for gRNA synthesis and concentrations of injected CRISPR/Cas9 ribonucleoprotein complexes. 


\section{Figure 1}

A

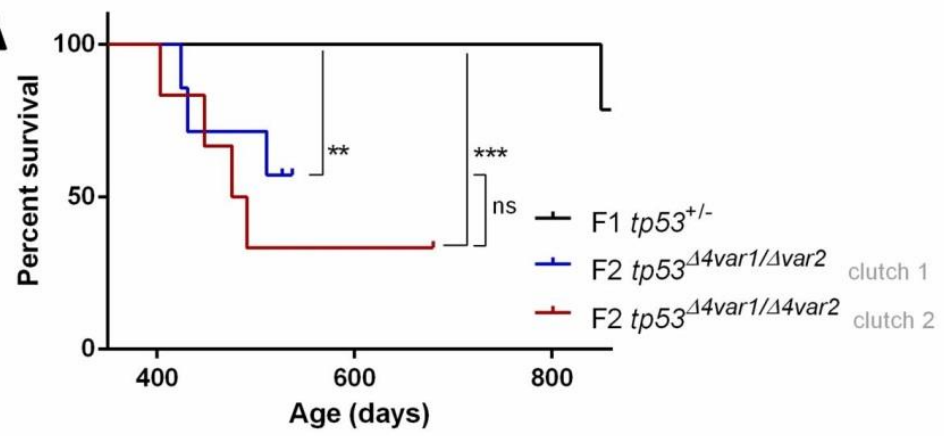

B

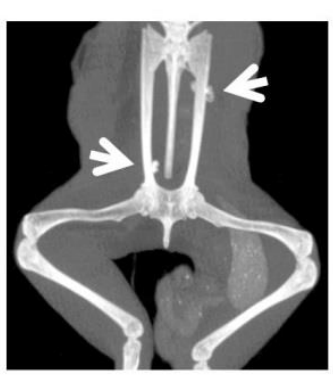

D

C

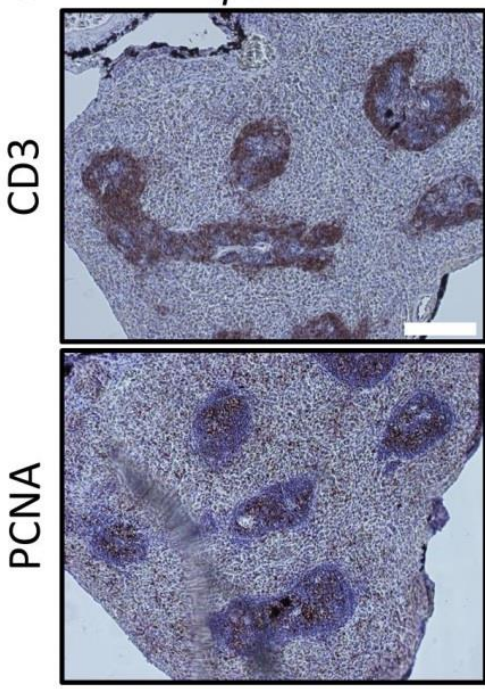

$\mathbf{F} \quad \mathrm{CD} 3$
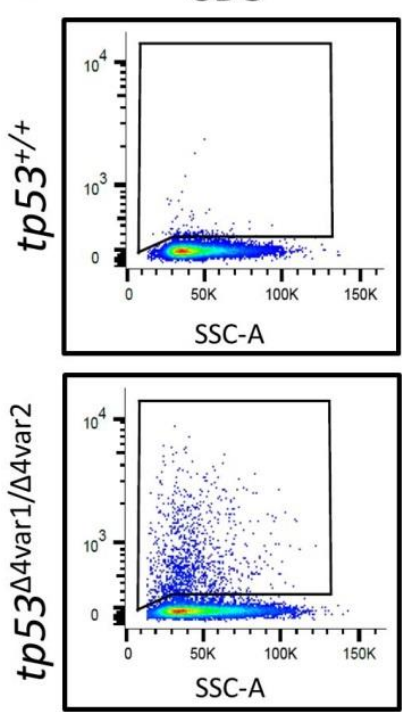

$\operatorname{tp5} 3^{\Delta 4 \operatorname{var} 1 / \Delta 4 \operatorname{var} 2}$
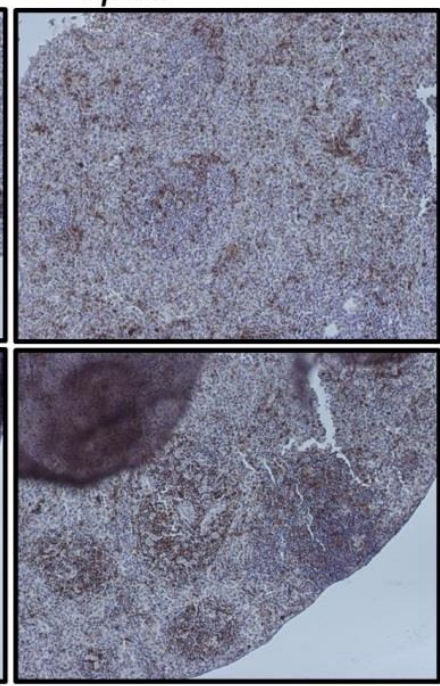

E

G
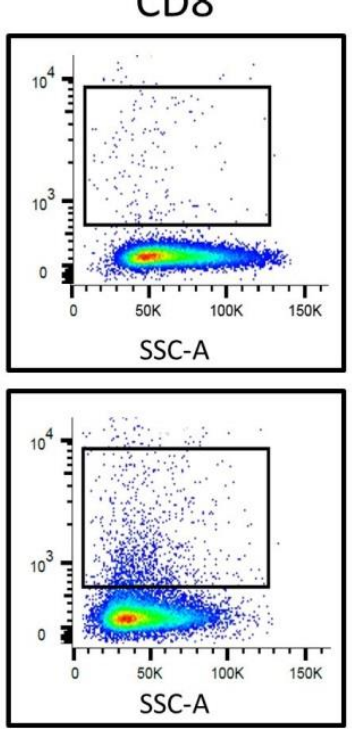

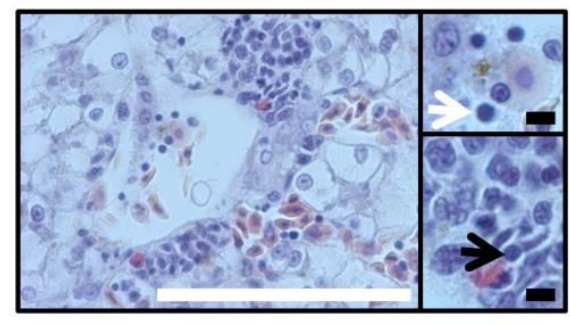

H

$\operatorname{tp} 53^{+/-}$

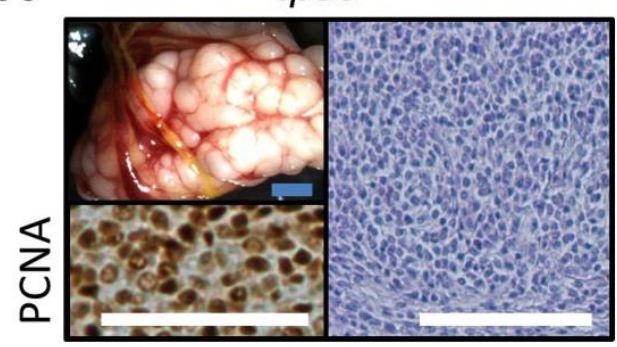




\section{$831 \quad$ Figure 2}

A
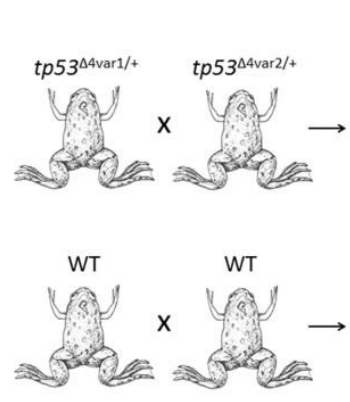

C

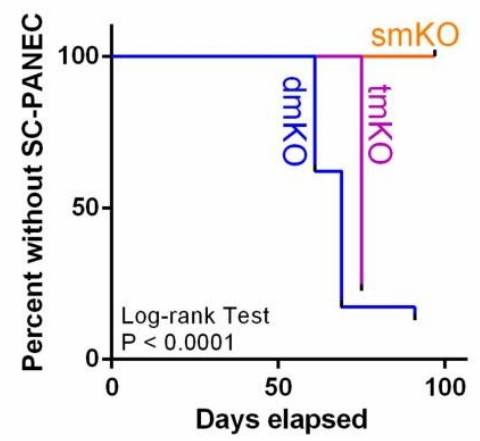

$\mathbf{F}$

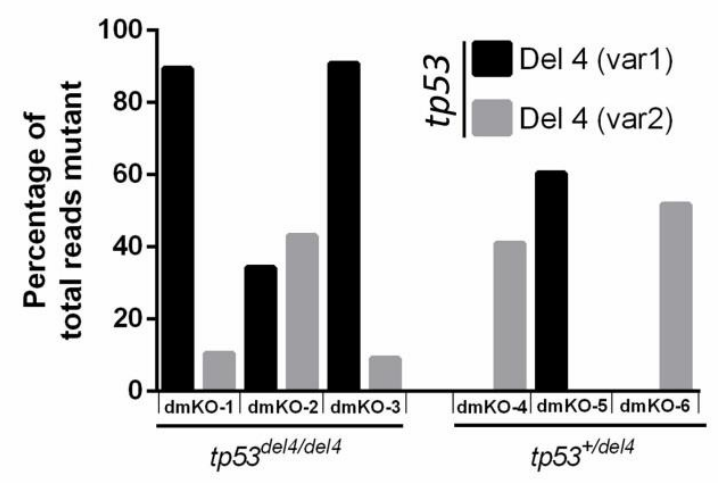

D

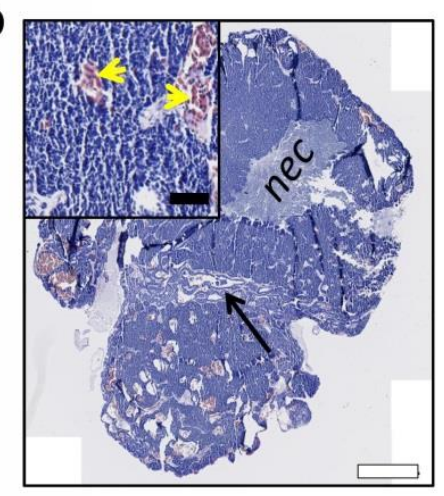

G
B

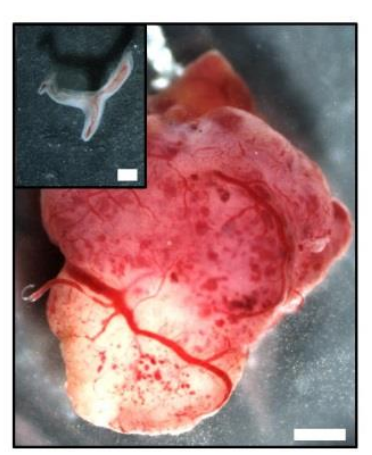

E

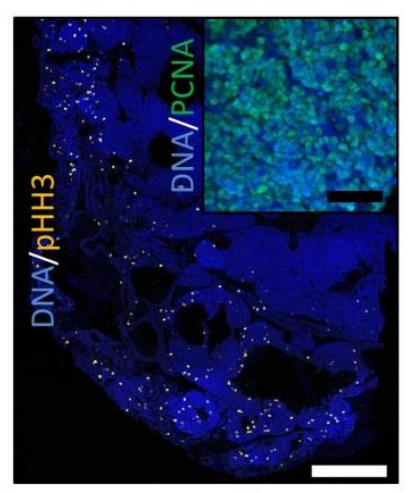

b1/rbl1 CRISPR/Cas9
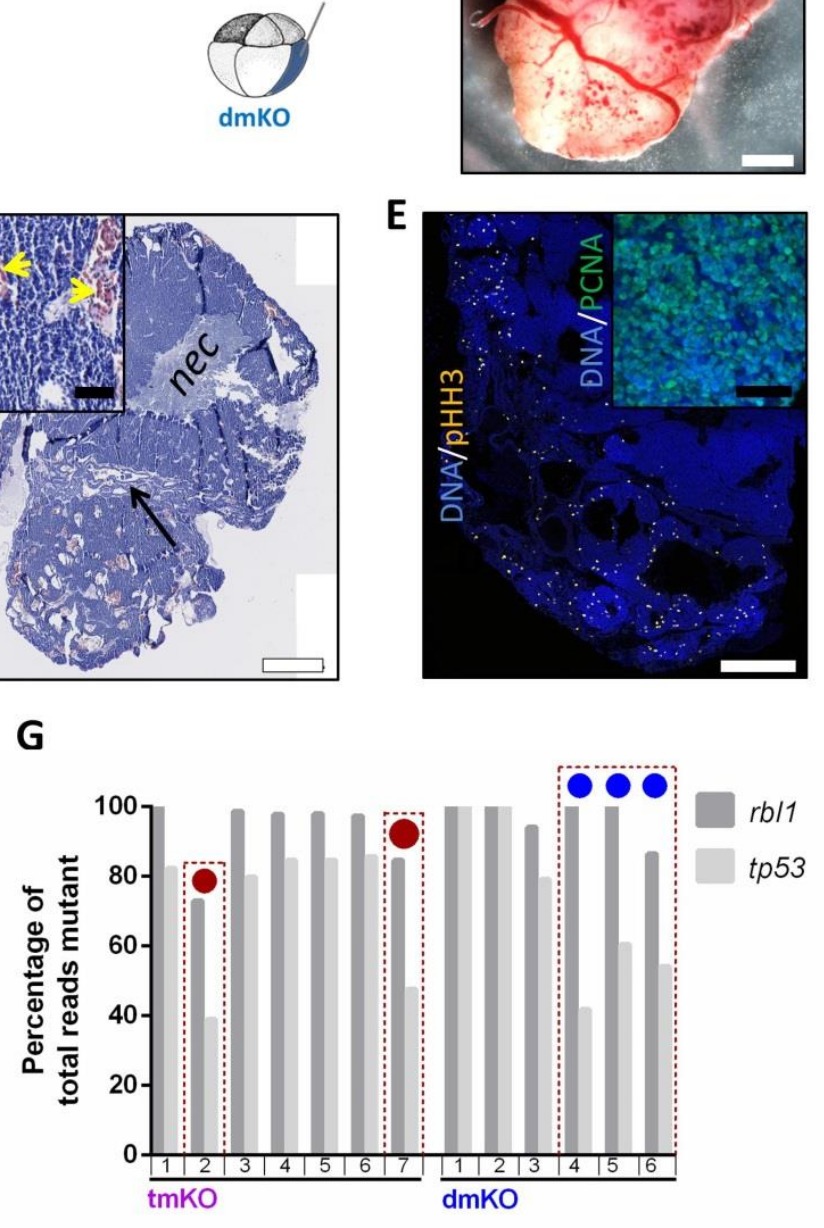


\section{Figure 3}

A

E

rb1/rbl1 CRISPR/Cas9 Right animal-dorsal

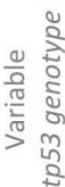

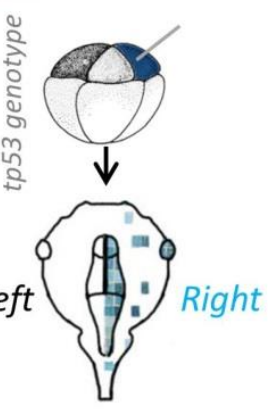

B C D
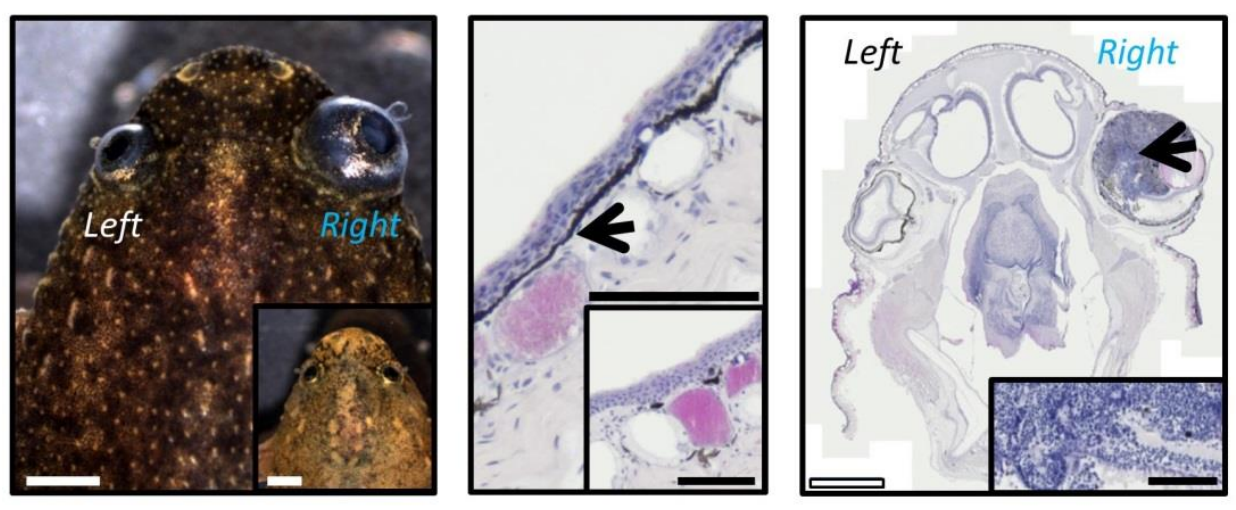

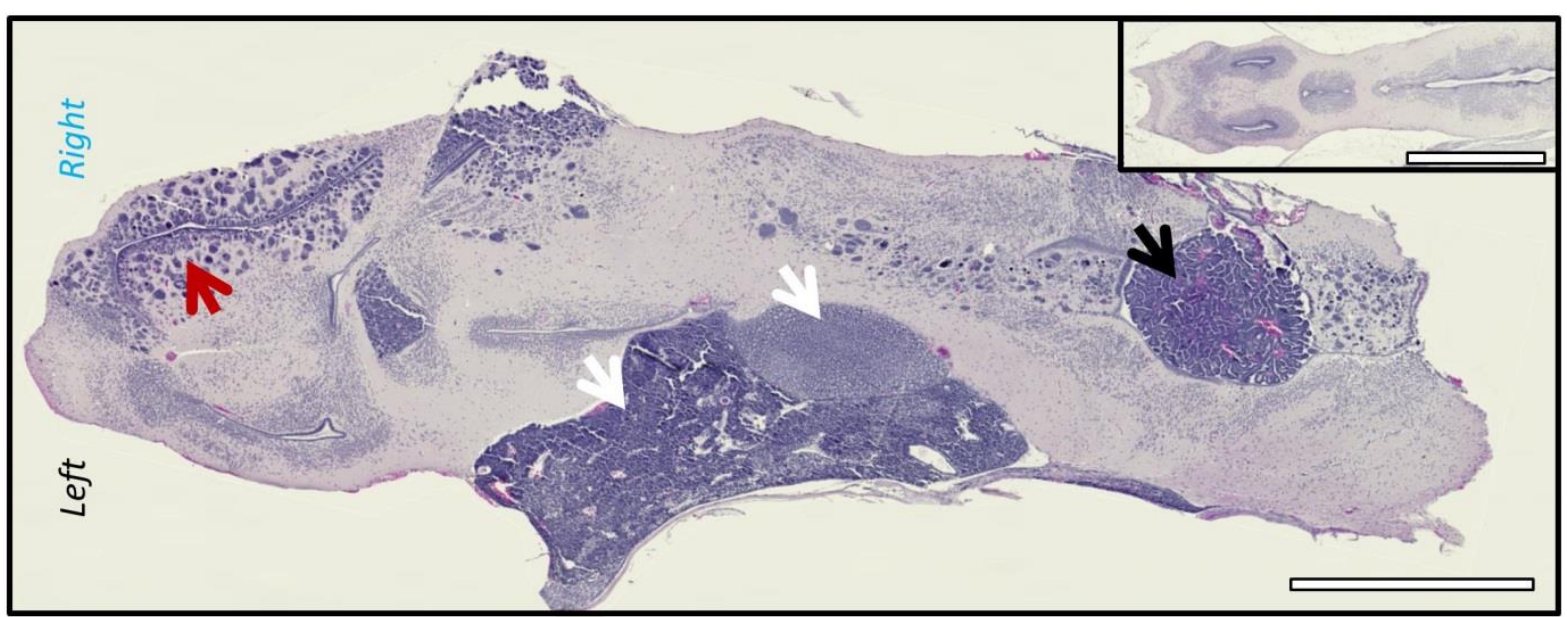


bioRxiv preprint doi: https://doi.org/10.1101/528299; this version posted January 23, 2019. The copyright holder for this preprint (which was not certified by peer review) is the author/funder. All rights reserved. No reuse allowed without permission.

Figure 4

A
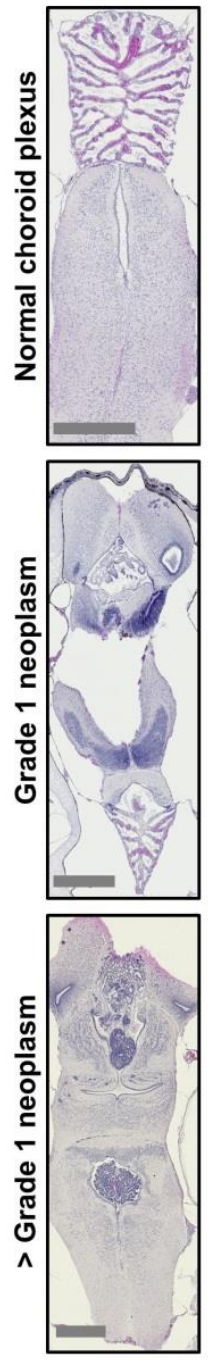
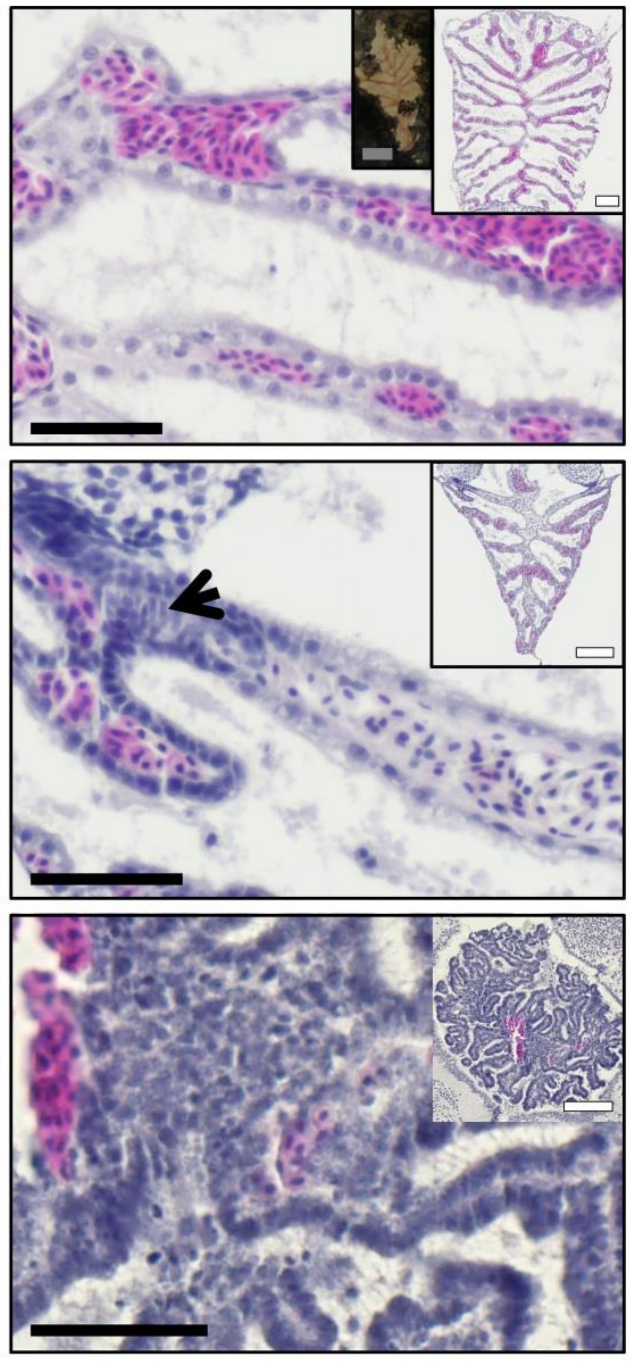

B

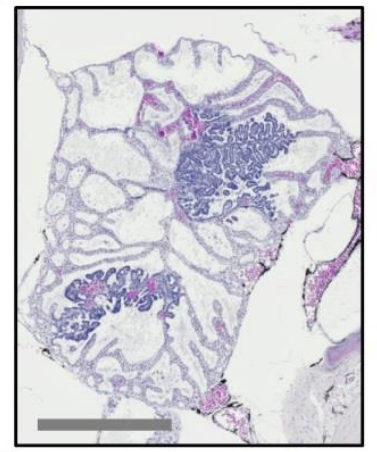

C

Choroid plexus neoplasm across tp53 genotypes
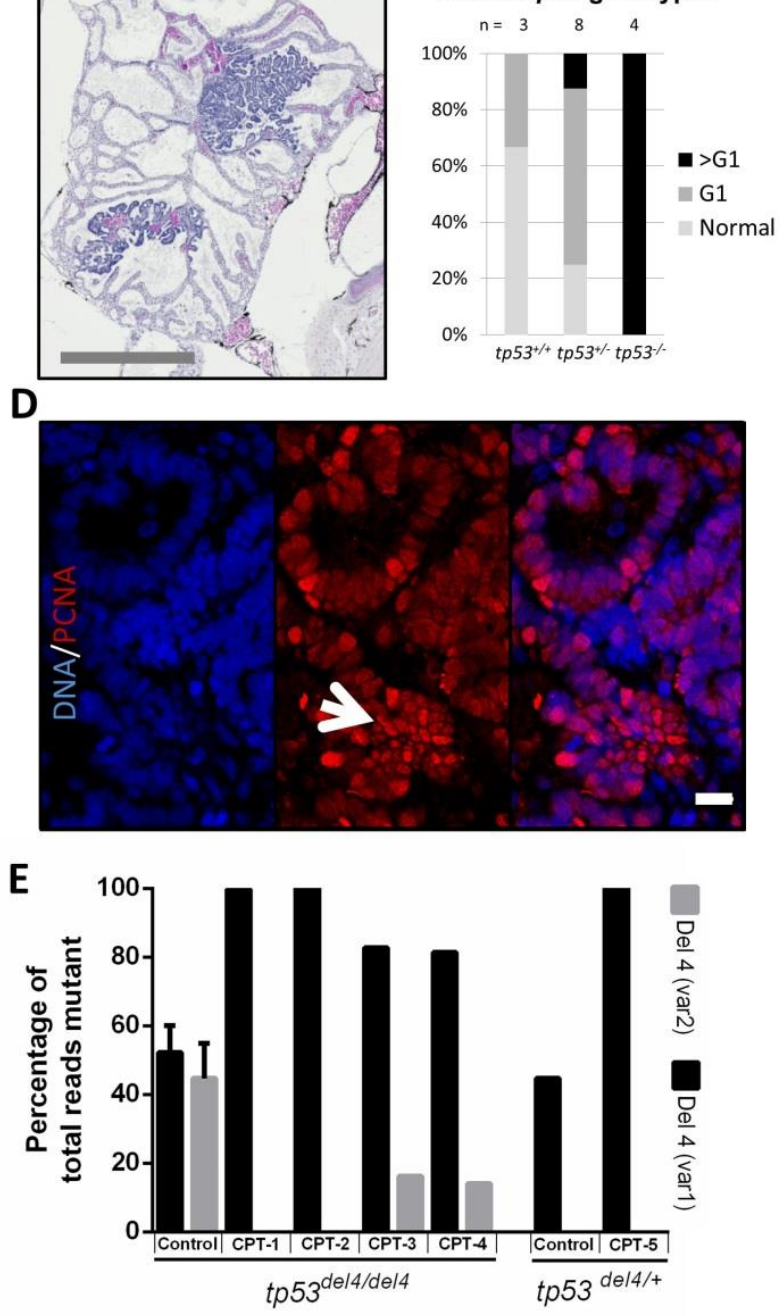


\section{$840 \quad$ Figure 5}

A Low-grade glioma

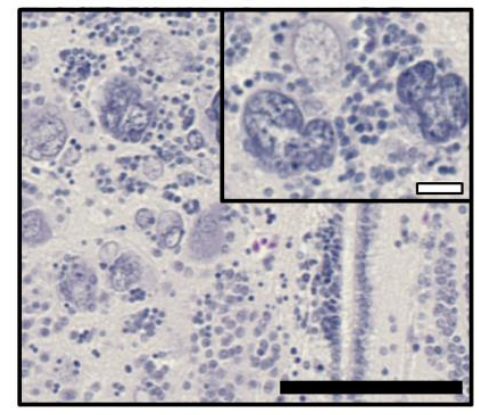

B

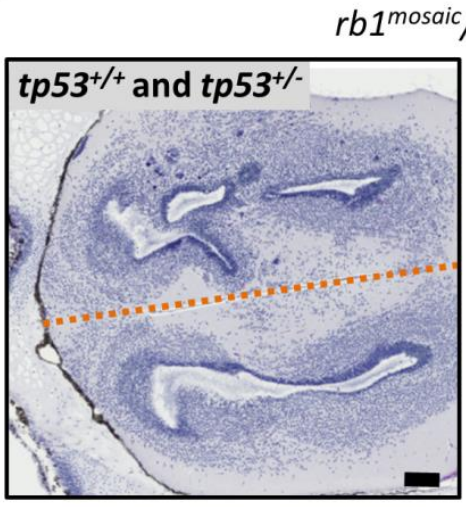

D

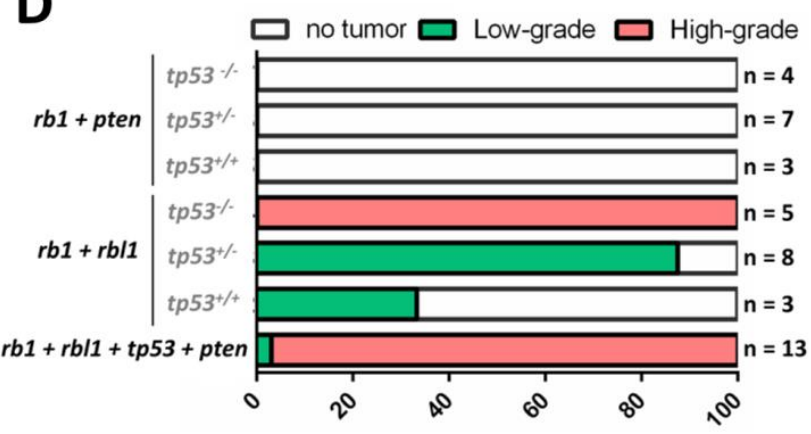

High-grade glioblastoma
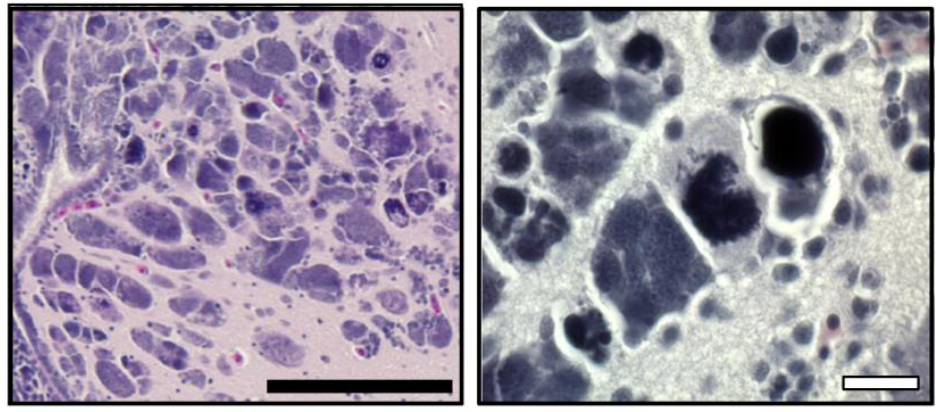

C $r b 1^{\text {mosaic }} / r b / 1^{\text {mosaic }} /$ tp53 $3^{\text {mosaic } / p^{\text {pten }} \text { mosaic }}$
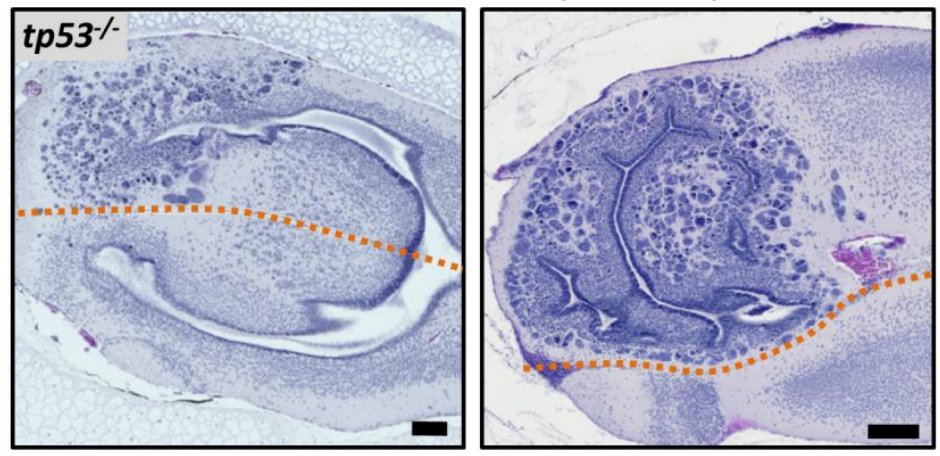

E

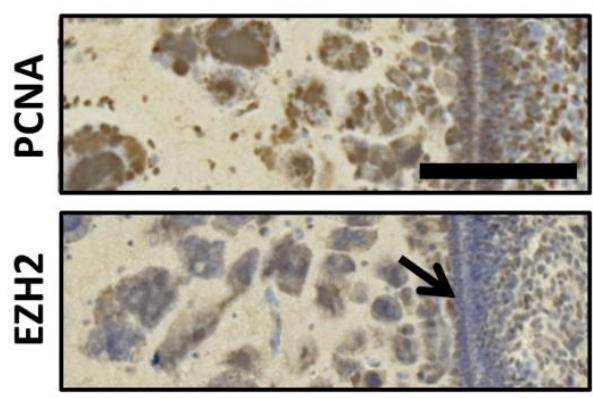

